\title{
APPLICATIONS OF $q$-LAGRANGE INVERSION TO BASIC HYPERGEOMETRIC SERIES
}

BY

\author{
IRA GESSEL $^{1}$ AND DENNIS STANTON ${ }^{2}$
}

\begin{abstract}
A family of $q$-Lagrange inversion formulas is given. Special cases include quadratic and cubic transformations for basic hypergeometric series. The $q$-analogs of the so-called "strange evaluations" are also corollaries. Some new RogersRamanujan identities are given. A connection between the work of Rogers and Andrews, and $q$-Lagrange inversion is stated.
\end{abstract}

1. Introduction. The Lagrange inversion formula solves the following problem: Given

$$
y=y(x)=b_{1} x+b_{2} x^{2}+\cdots, \quad b_{1} \neq 0,
$$

and

$$
f(x)=f_{0}+f_{1} x+f_{2} x^{2}+\cdots
$$

as formal power series in $x$, find the constants $a_{k}$ so that

$$
f(x)=a_{0}+a_{1} y+a_{2} y^{2}+\cdots .
$$

The special case $f(x)=x$ gives the inverse function of $y(x)$. Recently, $q$-analogs of this problem have been studied by Andrews [4], Gessel [23], and Garsia [22]. As Garsia shows, they are equivalent to finding the constants $a_{k}$ for

$$
f(x)=a_{0}+a_{1} y(x)+a_{2} y(x) y(x q)+\cdots .
$$

Unfortunately, there are very few examples for which this beautiful theory applies easily. (The case $y(x)=x /(1-x)$ works and was considered by Carlitz [15].) In this paper we give many examples of $q$-analogs of Lagrange inversion.

However, our examples do not satisfy (1.1). Instead, we concentrate on $q$-analogs of Lagrange inversion for $y=x /(1-x)^{b+1}$. We state here an example $(b=1)$ of such a $q$-Lagrange inversion. Throughout this paper we consider only formal power series in $x$ whose coefficients are rational functions of $q$. We use the customary notation, $(a)_{\infty}=\prod_{i=0}^{\infty}\left(1-a q^{i}\right)$ and $(a)_{k}=(a)_{\infty} /\left(a q^{k}\right)_{\infty}$.

Received by the editors March 3, 1982.

1980 Mathematics Subject Classification. Primary 33A35; Secondary 05A19, 10A45.

Key words and phrases. Lagrange inversion, basic hypergeometric series, Rogers-Ramanujan identities.

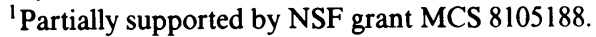

${ }^{2}$ Partially supported by NSF grant MCS 8102237 . 
THEOREM 1.2. Let $f(x)=\sum_{k=0}^{\infty} f_{k} x^{k}$. If

$$
f(x)=\frac{(A x)_{\infty}}{(x)_{\infty}} \sum_{k=0}^{\infty} a_{k} \frac{q^{-k^{2}} x^{k}}{(A x)_{k}(1-x / q) \cdots\left(1-x / q^{k}\right)}
$$

then $f_{n}=\sum_{k=0}^{n} B_{n k} a_{k}$, and vice versa, $a_{k}=\sum_{l=0}^{k} B_{k l}^{-1} f_{l}$, where

$$
B_{n k}=\frac{\left(A q^{2 k}\right)_{n-k}}{(q)_{n-k}} q^{-n k}
$$

and

$$
\left.B_{k l}^{-1}=\frac{(A q)_{2 k-1}\left(1-A q^{2 l}\right)}{(q)_{k-l}(A q)_{k+l}} q^{(k-l+1}\right)^{+k l}(-1)^{k-l} .
$$

This theorem explicitly solves for the $a_{k}$ 's in terms of the $f_{l}$ 's by giving the entries of the inverse matrix $B_{k l}^{-1}$. It is somewhat related to (1.1). If we put $A=1, y_{1}(x)=$ $1 /(1-x q)$, and $y_{2}(x)=1 /(1-x / q)$, then we are solving for the constants $a_{k}$ in

$$
f(x)=a_{0}+q^{-1} a_{1} x y_{1}(x) y_{2}(x)+q^{-4} a_{2} x^{2} y_{1}(x) y_{1}(x q) y_{2}(x) y_{2}\left(x q^{-1}\right)+\cdots .
$$

So the shifting in $q$ occurs in both directions, not just one as in (1.1).

Theorem 1.2 has some far-reaching consequences. It can be used to give a systematic approach to $q$-analogs of quadratic transformations. There is a scattering of such results in the literature $[3,16,27,29,33]$. However, the techniques are verifications by manipulating sums. With Theorem 1.2 we can systematically consider the "correct" series.

Perhaps the first $q$-quadratic transformation was Sears' [29, Equation (4.1)] $q$-ana$\log$ (see also [16]) of the well-poised ${ }_{3} F_{2}$ quadratic transformation. It follows from Theorem 1.2. Moreover, we derive Watson's transformation of a very well-poised ${ }_{8} \Phi_{7}[11$, Equation $8.5(2)]$ from this transformation. It is well known that Watson's transformation implies the Rogers-Ramanujan identities. Other q-quadratic transformations will imply identities that are similar to the Rogers-Ramanujan identities. Some of these are new, while others appear on Slater's list [31]. This is no accident. G. Andrews has commented [6] that much of Rogers' work was based upon the "Bailey transform". He then inverted the transform by finding the inverse of a certain matrix. That matrix is exactly our matrix $B$ in Theorem 1.2. So it is fair to say that Rogers' work was really a hidden special case of $q$-Lagrange inversion.

We have seen that Theorem 1.2 is the $q$-analog of Lagrange inversion for $x /(1-x)^{b+1}, b=1$. We also give $q$-analogs of Lagrange inversion for a general $b$ in Theorem 3.7. The theorems for $b=2,0,-2,-3$, and $1 / 2$ are also explicitly stated in $\S 3$. We concentrate on these values in $\$ \$ 5$ and 6 . In particular, we give more $q$-quadratic and $q$-cubic transformations in these cases.

In [24], we derived new evaluations of hypergeometric series by applying the Lagrange inversion formula to the $q=1$ version of these transformations. So in this 
paper we give $q$-analogs of the evaluations. For example, a $q$-analog of a cubic ${ }_{3} F_{2}$ transformation implies

$$
\begin{gathered}
\sum_{k=0}^{n} \frac{\left(q^{-n}\right)_{k}\left(A D^{2} q^{n+1 / 2}\right)_{k}\left(A / D^{2}\right)_{k}\left(A ; q^{1 / 2}\right)_{k}\left(q A / C ; q^{1 / 2}\right)_{k}\left(C q^{-1 / 2} / A ; q^{1 / 2}\right)_{k}\left(1-A q^{3 k / 2}\right)_{q}^{k / 2}}{(q)_{k}\left(A q^{n+1 / 2} ; q^{1 / 2}\right)_{k}\left(q^{-n} / D^{2} ; s q^{1 / 2}\right)_{k}\left(D^{2} q^{1 / 2} ; q^{1 / 2}\right)_{k}(C)_{k}\left(A^{2} q^{3 / 2} / C\right)_{k}(1-A)} \\
=\frac{\left(q^{1 / 2} A ; q^{1 / 2}\right)_{2 n}\left(C D^{2} / A\right)_{n}\left(D^{2} A q^{3 / 2} / C\right)_{n}}{\left(D^{2} q^{1 / 2} ; q^{1 / 2}\right)_{2 n}(C)_{n}\left(A^{2} q^{3 / 2} / C\right)_{n}}
\end{gathered}
$$

This is the $q$-analog of Equation (1.7) in [24]. The strange doubling of parameters there is now somewhat explained. The series (1.4) looks like a bibasic well-poised series. The first three pairs of numerator and denominator parameters multiply to $A q^{1 / 2}$, whereas the last three multiply to $A q$.

We could write (1.4) as a certain basic hypergeometric function $\left(a_{12} \Phi_{11}\right)$. However, we think that the series (1.4) is much more illuminating. For this paper we will use basic hypergeometric notation when it is not so clumsy. So in (1.4),

$$
\left(A ; q^{1 / 2}\right)_{k}=\prod_{j=0}^{k-1}\left(1-A q^{j / 2}\right)
$$

and

$$
(C)_{k}=\prod_{j=0}^{k-1}\left(1-C q^{j}\right),
$$

i.e. all bases are assumed to be $q$ unless otherwise stated.

We organize our paper in the following way. In $\$ 2$ we review Lagrange inversion for $x /(1-x)^{b+1}$. Then we are able to give the method for the $q$-analogs. They are stated in §3. Quadratic transformations and evaluations for Theorem 1.2 are given in §. A few more such examples for $b=0$ are in $\$ 5$. The other values of $b$ give new evaluations in $\S 6$. Special cases of transformations in $\$ 4$ give identities of the Rogers-Ramanujan type. They are stated in \$7. A connection between Theorem 1.2 and Andrews' paper on orthogonal polynomials is briefly stated in $\S 8$. Finally, prospects for further work are stated in $\$ 9$.

2. The method. Our method for finding $q$-analogs is based upon the following fact: the Lagrange inversion formula is equivalent to finding the inverse of an infinite lower triangular matrix [25]. To see this, put

$$
G_{k}(x)=y^{k}=\sum_{n=k}^{\infty} B_{n k} x^{n}, \quad B_{k k} \neq 0 .
$$

Then, the coefficient of $x^{n}$ in $f(x)=\sum_{k=0}^{\infty} a_{k} G_{k}(x)$ is

$$
f_{n}=\sum_{k=0}^{n} B_{n k} a_{k} \text {. }
$$


To solve for the $a_{k}$ 's in terms of the $f_{n}$ 's is equivalent to finding the inverse of the matrix $B$,

$$
a_{n}=\sum_{k=0}^{n} B_{n k}^{-1} f_{k}
$$

The Lagrange inversion formula solves for $B_{n k}^{-1}$ in terms of the coefficients $\left\{b_{1}, b_{2}, \ldots\right\}$ that define the power series for $y$. The generalized inversion problem defines $G_{k}(x)$ by (2.1) for some lower triangular matrix $B_{n k}$. Then one wants to find the inverse matrix $B_{n k}^{-1}$. This process gives our examples of $q$-Lagrange inversion.

First we consider the example of the usual Lagrange inversion formula upon which our $q$-analogs are based. We take

$$
f(x)=\sum_{k=0}^{\infty} f_{k} x^{k}
$$

and try to expand

$$
f(x)=(1-x)^{-a} \sum_{k=0}^{\infty} a_{k}\left[x /(1-x)^{b+1}\right]^{k} .
$$

So, the function $G_{k}(x)$ here is

$$
G_{k}(x)=x^{k}(1-x)^{-a-k(b+1)},
$$

which, for $a=0$, is $y^{k}, y=x /(1-x)^{b+1}$. Thus

$$
B_{n k}=\frac{(a+(b+1) k)_{n-k}}{(n-k) !} .
$$

(In this section we adopt the usual $q=1$ notation: $(A)_{n}=\Gamma(A+n) / \Gamma(A)$.) By the Lagrange inversion formula (see [33]), we see that $a_{n}$ is the coefficient of $x^{n}$ in $(1+b x)(1-x)^{a-1+n(b+1)} f(x)$, so

$$
B_{n k}^{-1}=\frac{(1-a-n(b+1))_{n-k-1}}{(n-k) !}(-a-(b+1) k) .
$$

To find a $q$-analog of (2.7) and (2.8), we consider the orthogonality relations

$$
\text { (2.9) } \begin{aligned}
\delta_{n l} & =\sum_{k=l}^{n} B_{n k}^{-1} B_{k l} \\
& =\sum_{k=l}^{n} \frac{(1-a-n(b+1))_{n-k-1}}{(n-k) !}[-a-(b+1) k] \frac{(a+(b+1) l)_{k-l}}{(k-l) !}, \\
\text { (2.10) } \delta_{n l} & =\sum_{k=l}^{n} B_{n k} B_{k l}^{-1} \\
& =\sum_{k=l}^{n} \frac{(a+(b+1) k)_{n-k}(1-a-(b+1) k)_{k-l-1}}{(n-k) !(k-l) !}[-a-(b+1) l] .
\end{aligned}
$$


The relation (2.9) can be proved by summing two ${ }_{2} F_{1}(1)$ 's. For the following values of $b,(2.10)$ is equivalent to

$$
\frac{(a)_{n+2 l}}{(n-l) !(a)_{3 l}} F_{2}\left[\begin{array}{c}
l-n, l+(n+a) / 2, l+(n+a+1) / 2 \\
(a+3 l+1) / 2,(a+3 l+2) / 2
\end{array} \mid \begin{array}{c}
1 \\
(b=2),
\end{array}\right.
$$

$$
\frac{(a)_{n+l}}{(n-l) !(a)_{2 l}}{ }_{2} F_{1}\left[\begin{array}{c|c}
l-n, a+n+l & \\
a+2 l+1 & 1
\end{array}\right]=\delta_{n l} \quad(b=1),
$$

$$
\frac{(a)_{n}}{(n-l) !(a)_{l}}{ }_{1} F_{0}\left[l-n \mid \begin{array}{l}
l-n \\
\end{array}\right]=\delta_{n l} \quad(b=0),
$$

$$
\frac{(a)_{n-l}}{(n-l) !}{ }_{2} F_{1}\left[\begin{array}{c|c}
l-n,-a \\
1-a-n+l
\end{array} \mid 1\right]=\delta_{n l} \quad(b=-1),
$$

$$
\left.\frac{(-1)^{n-l}(1+2 l-a-n)_{n-l}}{(n-l) !} F_{2}\left[\begin{array}{c}
l-n,(l-a) / 2,(l-a+1) / 2 \\
l+(1-a-n) / 2, l+(2-a-n) / 2
\end{array}\right] 1\right]=\delta_{n l}
$$

$$
\begin{aligned}
& \frac{(-1)^{n-l}(1-a-n)_{n+2 l}{ }_{4} F_{3}}{(n-l) !(1-a-n)_{3 l}} \\
& \qquad\left[\begin{array}{c}
l-n,(2 l-a) / 3,(2 l-a+1) / 3,(2 l-a+2) / 3 \\
l+(1-a-n) / 3, l+(2-a-n) / 3, l+(3-a-n) / 3
\end{array}\right]=\delta_{n l} \\
& (b=-3) .
\end{aligned}
$$

The binomial theorem gives (2.13), Vandermonde's theorem gives (2.12) and (2.14), Saalschütz' theorem [11, Equation 2.2(1)] gives (2.11) and (2.15), and the Saalschützian ${ }_{4} F_{3}$ transformation [11, Equation 7.2(1)] gives (2.16). Another case is

$$
\begin{gathered}
\frac{(a+3 l / 2)_{n-l}}{(n-l) !} F_{2}\left[\begin{array}{c|}
(l-n) / 2,(1+l-n) / 2, a+n+l / 2 \\
1 / 2, a+3 l / 2+1
\end{array}\right] \\
+(-a-3 l / 2) \frac{(a+3 l / 2+3 / 2)_{n-l-1}}{(n-l-1) !} F_{2} \\
\cdot\left[\begin{array}{c}
(1+l-n) / 2,(2+l-n) / 2, n+a+(l+1) / 2 \\
3 / 2, a+(3 l+3) / 2
\end{array}\right]=0
\end{gathered}
$$


for $l<n(b=1 / 2)$. Both of the ${ }_{3} F_{2}$ 's in (2.17) can be evaluated by Saalschütz' theorem.

We find the $q$-analogs of $B_{n k}$ by finding the $q$-analogs of (2.11)-(2.17). The $q$-analogs of (2.11)-(2.17) are easily written down by considering the $q$-analogs of the appropriate summation theorems (see [31, Appendix]). The $q$-analog of (2.9) is the sum of two terminating ${ }_{2} \Phi_{1}$ 's. It is

$$
\begin{aligned}
& \sum_{k=l}^{n} \frac{\left(q^{1-a-(b+1) n}\right)_{n-k-1}\left(q^{a+(b+1) l}\right)_{k-l}}{(q)_{n-k}(q)_{n-l}} q^{-(k-l)(n+n b+a)} \\
& \quad \cdot\left[\left(1-q^{-a-b l-l}\right)\left(1-q^{n-l}\right)-\left(1-q^{(n-l)(b+1)}\right)\left(1-q^{k-l}\right)\right]=0, \quad l<n .
\end{aligned}
$$

Nevertheless, we do not use (2.18) in this paper. In order to write (2.18) as an orthogonality for $B_{n k}$ and $B_{k l}^{-1}$, we need to separate the $n$ and $l$ dependence. We find it more convenient to use (2.11)-(2.17) for this purpose. Fields and Ismail [21] had previously given (2.9). They also considered inverting an infinite upper triangular matrix, but did not mention the connection with Lagrange inversion.

3. The $q$-analogs. In this section we state the $q$-analogs of Lagrange inversion based upon the $q$-analogs of (2.11)-(2.17). We do not give the tedious yet simple calculations that change those evaluations into a matrix orthogonality.

THEOREM $3.1\left(q\right.$-ANALOG OF LAGRANGE INVERSION FOR $\left.x /(1-x)^{3}\right)$. Let

$$
G_{k}(x)=\sum_{n=k}^{\infty} B_{n k} x^{n}
$$

where

$$
B_{n k}=\frac{\left(A q^{3 k / 2} ; q^{1 / 2}\right)_{n-k}}{(q)_{n-k}} q^{-n k}
$$

If $f(x)=\sum_{k=0}^{\infty} f_{k} x^{k}=\sum_{k=0}^{\infty} a_{k} G_{k}(x)$, then $f_{n}=\sum_{k=0}^{n} B_{n k} a_{k}$, and vice versa, $a_{k}=$ $\sum_{l=0}^{k} B_{k l}^{-1} f_{l}$, where

$$
B_{k l}^{-1}=\frac{\left(A q^{1 / 2} ; q^{1 / 2}\right)_{3 k-1}\left(1-A q^{3 l / 2}\right)}{(q)_{k-l}\left(A q^{1 / 2} ; q^{1 / 2}\right)_{2 k+l}} q^{\left({ }^{k-l+1}\right)+k l}(-1)^{k-l} .
$$

THEOREM $3.2\left(q\right.$-ANALOG OF LAGRANGE INVERSION FOR $\left.x /(1-x)^{2}\right)$. Let

$$
G_{k}(x)=\frac{(A x)_{\infty} q^{-k^{2}} x^{k}}{(x)_{\infty}(A x)_{k}(1-x / q) \cdots\left(1-x / q^{k}\right)}=\sum_{n=k}^{\infty} B_{n k} x^{n}
$$

where

$$
B_{n k}=\frac{\left(A q^{2 k}\right)_{n-k}}{(q)_{n-k}} q^{-k n} .
$$

If $f(x)=\sum_{k=0}^{\infty} f_{k} x^{k}=\sum_{k=0}^{\infty} a_{k} G_{k}(x)$, then $f_{n}=\sum_{k=0}^{n} B_{n k} a_{k}$, and vice versa, $a_{k}=$ $\sum_{l=0}^{k} B_{k l}^{-1} f_{l}$, where

$$
B_{k l}^{-1}=\frac{(A q)_{2 k-1}\left(1-A q^{2 l}\right)}{(q)_{k-l}(A q)_{k+l}} q^{\left(k-\frac{l+1}{2}\right)+k l}(-1)^{k-l} .
$$


THEOREM 3.3 ( $q$-ANALOG OF LAGRANGE INVERSION FOR $x /(1-x)$ ). Let

$$
G_{k}(x)=\frac{(A x)_{\infty} q^{-k^{2}} x^{k}}{(x)_{\infty}(1-x / q) \cdots\left(1-x / q^{k}\right)}=\sum_{n=k}^{\infty} B_{n k} x^{n}
$$

where

$$
B_{n k}=\frac{\left(A q^{k}\right)_{n-k}}{(q)_{n-k}} q^{-k n} .
$$

If $f(x)=\sum_{k=0}^{\infty} f_{k} x^{k}=\sum_{k=0}^{\infty} a_{k} G_{k}(x)$, then $f_{n}=\sum_{k=0}^{n} B_{n k} a_{k}$, and vice versa, $a_{k}=$ $\sum_{l=0}^{k} B_{k l}^{-1} f_{l}$, where

$$
\left.B_{k l}^{-1}=\frac{\left(A q^{l}\right)_{k-l}}{(q)_{k-l}} q^{(k-l+1}\right)+l k(-1)^{k-l}
$$

THEOREM 3.4 ( $q$-ANALOG OF LAGRANGE INVERSION FOR $x(1-x)$ ). Let $G_{k}(x)=$ $\sum_{n=k}^{\infty} B_{n k} x^{n}$, where

$$
B_{n k}=\frac{\left(A q^{k / 2} ; q^{-1 / 2}\right)_{n-k}}{(q)_{n-k}} q^{-n k} .
$$

If $f(x)=\sum_{k=0}^{\infty} f_{k} x^{k}=\sum_{k=0}^{\infty} a_{k} G_{k}(x)$, then $f_{n}=\sum_{k=0}^{n} B_{n k} a_{k}$, and vice versa, $a_{k}=$ $\sum_{l=0}^{k} B_{k l}^{-1} f_{l}$, where

$$
\left.B_{k l}^{-1}=\frac{\left(A q^{(k+1) / 2} ; q^{1 / 2}\right)_{k-l-1}}{(q)_{k-l}}\left(1-A q^{l / 2}\right)(-1)^{k-l} q^{(k-l+1}\right)+l k .
$$

TheOREM $3.5\left(q\right.$-ANALOG OF LAGRANGE INVERSION FOR $\left.x(1-x)^{2}\right)$. Let $G_{k}(x)=$ $\sum_{n=k}^{\infty} B_{n k} x^{n}$, where

$$
B_{n k}=\frac{\left(A q^{2 k / 3} ; q^{-1 / 3}\right)_{n-k}}{(q)_{n-k}} q^{-k n} .
$$

If $f(x)=\sum_{k=0}^{\infty} f_{k} x^{k}=\sum_{k=0}^{\infty} a_{k} G_{k}(x)$, then $f_{n}=\sum_{k=0}^{n} B_{n k} a_{k}$, and vice versa, $a_{k}=$ $\sum_{l=0}^{k} B_{k l}^{-1} f_{l}$, where

$$
B_{k l}^{-1}=\frac{\left(A q^{(2 k+1) / 3} ; q^{1 / 3}\right)_{k-l-1}}{(q)_{k-l}}\left(1-A q^{2 l / 3}\right) q^{\left({ }^{k-l+1}\right)+k l}(-1)^{k-l} .
$$

THEOREM $3.6\left(q\right.$-ANALOG OF LAGRANGE INVERSION FOR $\left.x /(1-x)^{3 / 2}\right)$. Let $G_{k}(x)$ $=\sum_{n=k}^{\infty} B_{n k} x^{n}$, where

$$
B_{n k}=\frac{\left(A q^{3 k / 2}\right)_{n-k}}{\left(q^{1 / 2} ; q^{1 / 2}\right)_{n-k}} q^{-k n / 2}
$$

If $f(x)=\sum_{k=0}^{\infty} f_{k} x^{k}=\sum_{k=0}^{\infty} a_{k} G_{k}(x)$, then $f_{n}=\sum_{k=0}^{n} B_{n k} a_{k}$, and vice versa, $a_{k}=$ $\sum_{l=0}^{k} B_{k l}^{-1} f_{l}$, where

$$
B_{k l}^{-1}=\frac{\left(A q^{3 k / 2-1} ; q^{-1}\right)_{k-l-1}}{\left(q^{1 / 2} ; q^{1 / 2}\right)_{k-l}}\left(1-A q^{3 l / 2}\right) q^{\left[\left({ }^{k-l+1}-1\right)+k l\right] / 2}(-1)^{k-l} .
$$

The matrices in Theorems 3.1-3.6 are so much alike that one could hope for a general theorem. Al-Salam and Verma [3] have given such a matrix. It is equivalent to the following theorem. 
THEOREM 3.7 ( $q$-ANALOG OF LAGRANGE INVERSION FOR $\left.x /(1-x)^{b+1}\right)$. Let $G_{k}(x)=\sum_{n=k}^{\infty} B_{n k} x^{n}$, where

$$
B_{n k}=\frac{\left(A p^{k} q^{k} ; p\right)_{n-k}}{(q)_{n-k}} q^{-n k} .
$$

If $f(x)=\sum_{k=0}^{\infty} f_{k} x^{k}=\sum_{k=0}^{\infty} a_{k} G_{k}(x)$, then $f_{n}=\sum_{k=0}^{n} B_{n k} a_{k}$, and vice versa, $a_{k}=$ $\sum_{l=0}^{k} B_{k l}^{-1} f_{l}$, where

$$
B_{k l}^{-1}=\frac{\left(A q^{k} p^{k-1} ; p^{-1}\right)_{k-l-1}}{(q)_{k-l}}\left(1-A p^{l} q^{l}\right) q^{k l+\left(c_{2}^{k-1+1}\right)}(-1)^{k-l} .
$$

Note that by choosing $p=q^{1 / b}$, we retain Theorems 3.1-3.6. If $A=q^{a / b}$, and $q \rightarrow 1$, we have

$$
G_{k}(x) \rightarrow x^{k}(1-x / b)^{-a-k(b+1)} .
$$

We have stated Theorems 3.1-3.6 because these are the special cases of Theorem 3.7 that give identities for basic hypergeometric series.

We did not state the formulas coming from the $q$-analog of (2.14). These are just multiplying $f(x)$ by $(x)_{\infty} /(A x)_{\infty}$. In Theorems 3.2 and 3.3 we used the $q$-binomial theorem to explicitly sum $G_{k}(x)=\sum_{n=k}^{\infty} B_{n k} x^{n}$. For example, in Theorem 3.2 we have

$$
G_{k}(x)=x^{k} q^{-k^{2}} \sum_{n=0}^{\infty} \frac{\left(A q^{2 k}\right)_{n}}{(q)_{n}}\left(x q^{-k}\right)^{n} .
$$

The $q$-binomial theorem implies that, as formal power series in $x$ with coefficients that are rational functions of $q$,

$$
G_{k}(x)=x^{k} q^{-k^{2}}\left(A x q^{k}\right)_{\infty} /\left(x q^{-k}\right)_{\infty} .
$$

However, we can also write

$$
G_{k}(x)=x^{k} q^{-k^{2}} \sum_{n=0}^{\infty} \frac{\left(A^{-1} q^{-2 k} ; q^{-1}\right)_{n}}{\left(q^{-1} ; q^{-1}\right)_{n}}\left(A x q^{k-1}\right)^{n}
$$

so that

$$
G_{k}(x)=x^{k} q^{-k^{2}}\left(x q^{-k-1} ; q^{-1}\right)_{\infty} /\left(A x q^{k-1} ; q^{-1}\right)_{\infty} .
$$

Equations (3.9) and (3.11) are not contradictory. They are merely two different ways to express $G_{k}(x)$ as a formal power series in $x$ with coefficients that are rational functions of $q$. This allows us the freedom to "change $q$ to $q^{-1}$ ". We have chosen (3.9) for Theorem 3.2.

There is a general theorem of which (3.8) and (3.10) are special cases. Let $\mathbf{C}[[x]]$ be the ring of formal power series in $x$ with coefficients in C. Similarly, define formal power series rings $\mathbf{C}(q)[[x]], \mathbf{C}((q))[[x]]$, and $\mathbf{C}\left(\left(q^{-1}\right)\right)[[x]]$, whose coefficients are rational functions of $q$, Laurent series in $q$, and Laurent series in $q^{-1}$, respectively. For the Laurent series in $q, \mathbf{C}((q))$, we allow only a finite number of negative powers 
of $q$. The coefficients in $\mathbf{C}\left(\left(q^{-1}\right)\right)$ are restricted similarly. Note that $\mathbf{C}(q) \subset \mathbf{C}((q)) \cap$ $\mathbf{C}\left(\left(q^{-1}\right)\right)$. Then for any $f(x) \in \mathbf{C}[[x]]$ with $f(0)=1$, we see that

$$
F(x)=\prod_{k=0}^{\infty} f\left(x q^{k}\right) \in \mathbf{C}(q)[[x]] .
$$

Next we state a theorem of Garsia [22, Proposition 2.2] which tells us how to interpret $F(x)$ as an element of $\mathbf{C}\left(\left(q^{-1}\right)\right)[[x]]$ via $\mathbf{C}(q)[[x]]$.

TheOREM 3.13. Let $f(x) \in \mathbf{C}[[x]]$ with $f(0)=1$. Then

$$
\prod_{k=0}^{\infty} f\left(x q^{k}\right) \in \mathbf{C}(q)[[x]] \text {. }
$$

Moreover, as an element of $\mathbf{C}\left(\left(q^{-1}\right)\right)[[x]]$,

$$
\prod_{k=0}^{\infty} f\left(x q^{k}\right)=1 / \prod_{k=1}^{\infty} f\left(x q^{-k}\right) \text {. }
$$

ProOF. Let $f(x)=\exp \left(\sum_{j=1}^{\infty} b_{j} x^{j}\right)$. Then

$$
\prod_{k=0}^{\infty} f\left(x q^{k}\right)=\exp \left(\sum_{j=1}^{\infty} b_{j} x^{j} /\left(1-q^{j}\right)\right) \in \mathbf{C}(q)[[x]] .
$$

The second statement follows from

$$
b_{j} x^{j} /\left(1-q^{j}\right)=-b_{j}\left(x q^{-1}\right)^{j} /\left(1-q^{-j}\right) .
$$

When necessary, we will freely use this $q \rightarrow q^{-1}$ idea.

In Theorems 3.1 and 3.4-3.6, we cannot express the function $G_{k}(x)$ as an infinite product. This is disappointing. However, we will find many new results from these theorems. Also in these cases $G_{k}(x)$ does not approach $x^{k}(1-x)^{-a-(b+1) k}$ as $q \rightarrow 1$. The limit is given in the discussion following Theorem 3.7. When $q=1$ the scaling factor of $b$ is not important.

Carlitz [15] had given Theorem 3.3 previously, although he stated it in another way.

For each of our theorems, we can state a natural "derivative form". For $q=1$ this is the expansion

$$
f(x)=(1+b x)(1-x)^{-a} \sum_{k=0}^{\infty} a_{k} x^{k} /(1-x)^{(b+1) k}
$$

The derivative of $x /(1-x)^{b+1}$ is $(1+b x) /(1-x)^{b+2}$. In [24] we showed that any expansion of type (2.5) naturally gives an expansion of type (3.14). We called the latter the companion formula. The $q$-analog of this procedure is to take the linear factor from $B_{k l}^{-1}$ and attach it to $B_{n k}$. We illustrate this technique on Theorem 3.2.

THEOREM 3.15 ( $q$-ANALOG OF DERIVATIVE FORM OF LAGRANGE INVERSION FOR $\left.x /(1-x)^{2}\right)$. Let

$$
G_{k}(x)=\frac{(A x)_{\infty}}{(x)_{\infty}}\left(1-q A x^{2}\right) \frac{q^{-k^{2}} x^{k}\left(1-A q^{2 k}\right)}{(A x)_{k+2}(1-x / q) \cdots\left(1-x / q^{k}\right)}=\sum_{n=k}^{\infty} C_{n k} x^{n}
$$


where

$$
C_{n k}=\frac{\left(A q^{2 k}\right)_{n-k}}{(q)_{n-k}} q^{-k n}\left(1-A q^{2 n}\right) .
$$

If $f(x)=\sum_{k=0}^{\infty} f_{k} x^{k}=\sum_{k=0}^{\infty} a_{k} G_{k}(x)$, then $f_{n}=\sum_{k=0}^{n} C_{n k} a_{k}$, and vice versa, $a_{k}=$ $\sum_{l=0}^{k} C_{k l}^{-1} f_{l}$, where

$$
\left.C_{k l}^{-1}=\frac{(A q)_{2 k-1}(-1)^{k-l}}{(q)_{k-l}(A q)_{k+l}} q^{(k-l+1}\right)+l k .
$$

As a prelude to $\S \S 4-6$, we give an application of Theorem 3.7 to Abel's [2] generalization of the binomial theorem. If we let $b \rightarrow \infty(p=1)$ in Theorem 3.7, we find a $q$-analog of $G_{k}(x)=x^{k} e^{k \beta x}$. Lagrange inversion for $G_{k}(x)=x^{k} e^{k \beta x}$ yields Abel's formula. So Theorem 3.7 should give a $q$-analog. First we review the $q=1$ case.

If $G_{k}(x)=x^{k} e^{k \beta x}$, we have

$$
B_{n k}=\frac{(\beta k)^{n-k}}{(n-k) !}
$$

and

$$
B_{k l}^{-1}=\frac{(-l \beta)(-k \beta)^{k-l-1}}{(k-l) !}
$$

The choices of

$$
f_{n}=\frac{\alpha^{n}}{n !}
$$

and

$$
a_{k}=\frac{\alpha(\alpha-\beta k)^{k-1}}{k !}
$$

give [1]

$$
e^{\alpha x}=\sum_{k=0}^{\infty} \frac{\alpha(\alpha-\beta k)^{k-1}}{k !}\left(x e^{\beta x}\right)^{k}
$$

and

$$
\frac{\alpha^{n}}{n !}=\sum_{k=0}^{n} \frac{\alpha(\alpha-\beta k)^{k-1}(\beta k)^{n-k}}{k !(n-k) !} .
$$

If we multiply $e^{\alpha x} \cdot e^{\gamma x}$ and use (3.20), we find one form of Abel's formula [2],

$$
\frac{(\alpha+\gamma)(\alpha+\gamma-\beta n)^{n-1}}{n !}=\sum_{k=0}^{n} \frac{\alpha(\alpha-\beta k)^{k-1} \gamma(\gamma-\beta(n-k))^{n-k-1}}{k !(n-k) !}
$$

For the $q$-analog, we put $p=1$ in Theorem 3.7 and choose

$$
f_{l}=\frac{B^{l}}{(q)_{l}}
$$


Then the $q$-binomial theorem implies

$$
a_{k}=\frac{\left(B q /\left(1-A q^{k}\right)\right)_{k-1}}{(q)_{k}}\left(1-A q^{k}\right)^{k-1} q^{\left({ }^{k+1}\right)}(-1)^{k}(1-A-B) .
$$

Also, it is easy to see that

$$
G_{k}(x)=q^{-k^{2}} x^{k} /\left(\left(1-A q^{k}\right) x\right)_{\infty}
$$

\section{So}

$$
\frac{1}{(B x)_{\infty}}=\sum_{k=0}^{\infty} \frac{\left(B q /\left(1-A q^{k}\right)\right)_{k-1}\left(1-A q^{k}\right)^{k-1}}{(q)_{k}\left(\left(1-A q^{k}\right) x\right)_{\infty}}(1-A-B)(-x)^{k} q^{-\left(\begin{array}{c}
k \\
2
\end{array}\right)} .
$$

This is a $q$-analog of (3.20) in the following way: Put $A=q^{a}$ and $B=\left(1-q^{b}\right)$, and let $q \rightarrow 1$. We find

$$
e^{b x}=\sum_{k=0}^{\infty} \frac{(b-a)(b-a-k)^{k-1}}{k !} x^{k} e^{k x+a x} .
$$

F. H. Jackson's [26, Equation (5)] is closely related to (3.26). The $q$-analog of (3.21) is

$$
\frac{B^{n}}{(q)_{n}}=\sum_{k=0}^{n} \frac{\left(B q /\left(1-A q^{k}\right)\right)_{k-1}}{(q)_{k}(q)_{n-k}}\left(1-A q^{k}\right)^{n-1}(1-A-B) q^{-n k+\left(\begin{array}{c}
k+1 \\
2
\end{array}\right)}(-1)^{k}
$$

To find a $q$-analog of one form of (3.22), we consider

$$
\sum_{n=0}^{n} C_{N n} f_{n}=\sum_{n, k} C_{N n} B_{n k} a_{k} \text {. }
$$

The choice of

$$
C_{N n}=\left(q^{-N}\right)_{n} C^{n}
$$

implies

$$
\begin{aligned}
& \frac{\left(C B q^{-N}\right)_{N}}{(q)_{N}} \\
& =\sum_{k=0}^{N} \frac{(1-A-B)\left(1-A q^{k}-B q\right) \cdots\left(1-A q^{k}-B q^{k-1}\right)\left(1-C q^{-N}\left(1-A q^{k}\right)\right) \cdots\left(1-C q^{-k-1}\left(1-A q^{k}\right)\right)}{(q)_{k}(q)_{N-k}} \\
& \quad C^{k} q^{-N k} .
\end{aligned}
$$

This differs from (3.22) because there are $N-k$ (not $N-k-1$ ) terms in the second product. We have chosen it because it implies F. H. Jackson's formula [26, Equation (2)].

It is clear that other variations are possible. We do not claim that any of these formulas are deep. They are all easy to prove by $q$-difference operators. However, it is interesting that the family of $q$-Lagrange inversion formulas led directly to this example. Also, the same techniques will be applied to find some important results later. 
4. Evaluations and quadratic transformations. We can choose $a_{k}$ in Theorem 3.2 as we wish. For any choice of $a_{k}$ for which we can evaluate

$$
f_{n}=\sum_{k=0}^{n} B_{n k} a_{k}
$$

we have a quadratic transformation for $f(x)$. We also find a dual evaluation

$$
a_{k}=\sum_{l=0}^{k} B_{k l}^{-1} f_{l}
$$

by $q$-Lagrange inversion. Of course these remarks apply to any of the theorems stated in §3. However, in this section we restrict our attention to Theorem 3.2. Andrews [6] had given the dual identities in this case but not the related transformations.

We simplify the right-hand side of (4.1) to

$$
f_{n}=\frac{(A)_{n}}{(q)_{n}} \sum_{k=0}^{n} \frac{\left(q^{-n}\right)_{k}\left(A q^{n}\right)_{k}}{(A)_{2 k}} q^{-\left(\begin{array}{c}
k \\
2
\end{array}\right)}(-1)^{k} a_{k} .
$$

So we choose $a_{k}$ (which is independent of $n$ ) so that (4.3) is evaluable.

As our first example we show that Sears' well-poised ${ }_{3} \Phi_{2}$ quadratic transformation [29] implies Watson's transformation of an ${ }_{8} \Phi_{7}$ into a ${ }_{4} \Phi_{3}$. We take

$$
a_{k}=\frac{(B)_{k}(A)_{2 k} q^{\left(\frac{k}{2}\right)+k}(-1)^{k}}{(q)_{k}(C)_{k}(q A B / C)_{k}}
$$

so that

$$
f_{n}=\frac{(A)_{n}(q A / C)_{n}(C / B)_{n} B^{n}}{(q)_{n}(C)_{n}(q A B / C)_{n}}
$$

by the $q$-analog of Saalschütz' theorem [30]. The result [29] is

$$
{ }_{3} \Phi_{2}\left[\begin{array}{c}
A, q A / C, C / B \\
C, q A B / C
\end{array} \mid q ; B x\right]=\frac{(A x)_{\infty}}{(x)_{\infty}} \sum_{k=0}^{\infty} \frac{(B)_{k}(A)_{2 k} q^{k}}{(q)_{k}(C)_{k}(q A B / C)_{k}(A x)_{k}(q / x)_{k}} .
$$

Again we caution that (4.6) is true only as a formal power series in $x$. The dual identity is equivalent to

$$
{ }_{6} \Phi_{5}\left[\begin{array}{c}
A,(A q)^{1 / 2},-(A q)^{1 / 2}, q A / C, C / B, q^{-n} \\
A^{1 / 2},-A^{1 / 2}, C, q A B / C, A q^{n+1}
\end{array} \mid q ; B q^{n}\right]=\frac{(B)_{n}(A q)_{n}}{(C)_{n}(q A B / C)_{n}}
$$

the very well-poised ${ }_{6} \Phi_{5}$ evaluation. Watson's transformation follows if we put

$$
C_{m n}=\frac{\left(1-A q^{2 n}\right)(D)_{n}\left(q^{-m}\right)_{n}(E)_{n}}{(1-A)(q A / D)_{n}\left(A q^{m+1}\right)_{n}(q A / E)_{n}}\left(\frac{A q^{m+1}}{D E}\right)^{n},
$$

form

$$
\sum_{n=0}^{m} C_{m n} f_{n}=\sum_{n=0}^{m} C_{m n} \sum_{k=0}^{n} B_{n k} a_{k}
$$


interchange the $n$ and $k$ sums, and evaluate the resulting $n$ sum by (4.7). This proof is not new. It is essentially Bailey's proof of boosting an evaluation to a transformation [13]. Andrews [6] gave a simplified related proof using the matrix $B$. However, the interpretation of this transformation by $q$-Lagrange inversion is new. In [24] we proved the $q=1$ version by multiplying two ${ }_{3} F_{2}$ quadratic transformations together. This $q$-analog is really the same. We could have written $C_{m n}$ as the coefficient of $x^{m-n}$ in the companion transformation to (4.6). So the left-hand side of (4.9) is the coefficient of $x^{m}$ in the product of two quadratic transformations.

Another choice is

$$
a_{k}=\frac{(A)_{2 k}\left(C ; q^{2}\right)_{k} q^{\left(\frac{k}{2}\right)+k}(-1)^{k}}{(q)_{k}\left(A q ; q^{2}\right)_{k}(C)_{k}}
$$

Then a $q$-analog of Watson's theorem [5, Theorem 1] gives

$$
f_{n}= \begin{cases}0, & n \text { odd }, \\ \frac{\left(A ; q^{2}\right)_{N}\left(A q / C ; q^{2}\right)_{N}}{\left(q^{2} ; q^{2}\right)_{N}\left(C q ; q^{2}\right)_{N}} C^{N}, & n=2 N \text { even }\end{cases}
$$

The transformation is

$$
\begin{aligned}
{ }_{2} \Phi_{1}\left[\begin{array}{c}
A, A q / C \\
C q
\end{array}\right. & \left.\mid q^{2} ; C x^{2}\right] \\
& =\frac{(A x)_{\infty}}{(x)_{\infty}} \sum_{k=0}^{\infty} \frac{\left(A ; q^{2}\right)_{k}\left(C ; q^{2}\right)_{k} q^{-\left(\frac{k}{2}\right)}(-x)^{k}}{(q)_{k}(C)_{k}(A x)_{k}(1-x / q) \cdots\left(1-x / q^{k}\right)} .
\end{aligned}
$$

This transformation was given by Verma [32]. The $C=0$ and $C \rightarrow \infty$ special cases of (4.12) are interesting. They are

$$
\begin{aligned}
\sum_{N=0}^{\infty} \frac{\left(A ; q^{2}\right)_{N}}{\left(q^{2} ; q^{2}\right)_{N}}\left(-q A x^{2}\right)^{N} q^{2\left(\frac{N}{2}\right)} \\
\quad=\frac{(A x)_{\infty}}{(x)_{\infty}} \sum_{k=0}^{\infty} \frac{\left(A ; q^{2}\right)_{k} q^{-\left(\frac{k}{2}\right)}(-x)^{k}}{(q)_{k}(A x)_{k}(1-x / q) \cdots\left(1-x / q^{k}\right)}
\end{aligned}
$$

and

$$
\sum_{N=0}^{\infty} \frac{\left(A ; q^{2}\right)_{N}}{\left(q^{2} ; q^{2}\right)_{N}} q^{-N^{2}}\left(-x^{2}\right)^{N}=\frac{(A x)_{\infty}}{(x)_{\infty}} \sum_{k=0}^{\infty} \frac{\left(A ; q^{2}\right)_{k}(-x)^{k}}{(q)_{k}(A x)_{k}(1-x / q) \cdots\left(1-x / q^{k}\right)}
$$

Both (4.13) and (4.14) are $q$-analogs of

$$
\left(1+x^{2}\right)^{-A}=(1-x)^{-2 A}\left(1+2 x /(1-x)^{2}\right)^{-A} .
$$

The dual evaluation to (4.11) is again a special case of (4.7). 
If we take $A=q$ and

$$
a_{k}=\frac{(A)_{2 k}\left(C ; q^{2}\right)_{k} q^{\left(\frac{k}{2}\right)+k}(-1)^{k}}{\left(q^{2} ; q^{2}\right)_{k}(E)_{k}(C q / E)_{k}},
$$

then the $q$-analog of Whipple's theorem [5, Theorem 2] gives

$$
f_{n}= \begin{cases}C^{N+1} \frac{\left(q / E ; q^{2}\right)_{N+1}\left(E / C ; q^{2}\right)_{N+1}}{\left(E ; q^{2}\right)_{N+1}\left(C q / E ; q^{2}\right)_{N+1}}, & n=2 N+1 \text { odd, } \\ C^{N} \frac{\left(q^{2} / E ; q^{2}\right)_{N}\left(q E / C ; q^{2}\right)_{N}}{\left(q E ; q^{2}\right)_{N}\left(C q^{2} / E ; q^{2}\right)_{N}}, & n=2 N \text { even. }\end{cases}
$$

The transformation is

$$
\begin{aligned}
& \sum_{N=0}^{\infty} \frac{\left(q / E ; q^{2}\right)_{N+1}\left(E / C ; q^{2}\right)_{N+1}}{\left(E ; q^{2}\right)_{N+1}\left(C q / E ; q^{2}\right)_{N+1}} C^{N+1} x^{2 N+1} \\
& +\sum_{N=0}^{\infty} \frac{\left(q^{2} / E ; q^{2}\right)_{N}\left(q E / C ; q^{2}\right)_{N}\left(C x^{2}\right)^{N}}{\left(q E ; q^{2}\right)_{N}\left(C q^{2} / E ; q^{2}\right)_{N}} \\
& =\frac{1}{1-x} \sum_{k=0}^{\infty} \frac{\left(q ; q^{2}\right)_{k}\left(C ; q^{2}\right)_{k} q^{-\left(\begin{array}{l}
k \\
2
\end{array}\right)}(-x)^{k}}{(E)_{k}(C q / E)_{k}(1-x / q) \cdots\left(1-x / q^{k}\right)} .
\end{aligned}
$$

This is the $q$-analog of a formula of Bailey [12, Equation (4.13)]. We do not give the dual evaluation.

Of course we can use the same idea to choose $f_{l}$ and find $a_{k}$. We have

$$
a_{k}=\frac{\left.(A q)_{2 k-1} q^{\left({ }_{2}+1\right.}\right)}{(q)_{k}(A q)_{k}} \sum_{l=0}^{k} \frac{\left(q^{-k}\right)_{l}}{\left(A q^{k+1}\right)_{l}} q^{k l}(-1)^{k} f_{l}\left(1-A q^{2 l}\right) .
$$

If we take

$$
f_{l}=\frac{(1+\sqrt{A})\left(A^{1 / 2} ; q^{1 / 2}\right)_{l}\left(B ; q^{1 / 2}\right)_{l}}{\left(1+\sqrt{A} q^{l}\right)\left(q^{1 / 2} ; q^{1 / 2}\right)_{l}\left(q^{1 / 2} A^{1 / 2} / B ; q^{1 / 2}\right)_{l}}\left(-q^{1 / 2} A^{1 / 2} / B\right)^{l}
$$

then the right-hand side of (4.18) can be evaluated by (4.7) with base $q^{1 / 2}$ to obtain

$$
a_{k}=\frac{(A)_{2 k}\left(-q^{1 / 2} A^{1 / 2} / B ; q^{1 / 2}\right)_{2 k}}{(q)_{k}\left(A q / B^{2}\right)_{k}\left(-q^{1 / 2} A^{1 / 2} ; q^{1 / 2}\right)_{2 k}} q^{\left({ }_{2}^{+1}\right)}(-1)^{k} .
$$

The transformation is

$$
\begin{aligned}
& \sum_{l=0}^{\infty} \frac{\left(A^{1 / 2} ; q^{1 / 2}\right)_{l}\left(B ; q^{1 / 2}\right)_{l}(1+\sqrt{A})}{\left(q^{1 / 2} ; q^{1 / 2}\right)_{l}\left(q^{1 / 2} A^{1 / 2} / B ; q^{1 / 2}\right)_{l}\left(1+\sqrt{A} q^{l}\right)}\left(-q^{1 / 2} A^{1 / 2} x / B\right)^{\prime} \\
& \quad=\frac{(A x)_{\infty}}{(x)_{\infty}} \sum_{k=0}^{\infty} \frac{(A)_{2 k}\left(-q^{1 / 2} A^{1 / 2} / B ; q^{1 / 2}\right)_{2 k} q^{-\left(\frac{k}{2}\right)}(-x)^{k}}{(q)_{k}\left(A q / B^{2}\right)_{k}(A x)_{k}(1-x / q) \cdots\left(1-x / q^{k}\right)\left(-q^{1 / 2} A^{1 / 2} ; q^{1 / 2}\right)_{2 k}}
\end{aligned}
$$


The dual evaluation is equivalent to

$$
\begin{aligned}
\left.{ }_{4} \Phi_{3}\left[\begin{array}{c}
q^{-n}, A q^{n},-A^{1 / 2} q^{1 / 2} / B,-A^{1 / 2} q / B \\
\\
A q / B^{2},-A^{1 / 2} q^{1 / 2},-A^{1 / 2} q
\end{array}\right] q ; q\right] \\
\quad=\frac{\left(-q^{1 / 2} ; q^{1 / 2}\right)_{n}\left(B ; q^{1 / 2}\right)_{n}(1+\sqrt{A})}{\left(-A^{1 / 2} ; q^{1 / 2}\right)_{n}\left(q^{1 / 2} A^{1 / 2} / B ; q^{1 / 2}\right)_{n}\left(1+\sqrt{A} q^{n}\right)}\left(-q^{1 / 2} A^{1 / 2} / B\right)^{n} .
\end{aligned}
$$

Andrews had previously given (4.22) in [6, Equation (4.3)]. His identities [6, Equations (4.5) and (4.7)] also follow in this way.

For [6, Equation (4.5)] we take

$$
f_{l}=\frac{\left(A^{1 / 3} ; q^{1 / 3}\right)_{l}(1-A)\left(1-A^{1 / 3} q^{2 l / 3}\right)}{\left(q^{1 / 3} ; q^{1 / 3}\right)_{l}\left(1-A q^{2 l}\right)\left(1-A^{1 / 3}\right)}(A q)^{l / 3}
$$

then again the right-hand side of (4.18) can be evaluated by (4.7) with base $q^{1 / 3}$ to obtain

$$
a_{k}=\frac{(1-A)\left(A^{1 / 3} q^{1 / 3} ; q^{1 / 3}\right)_{3 k} q^{\left(k_{2}^{k+1}\right)}(-1)^{k}}{\left(1-A q^{2 k}\right)(q)_{k}}
$$

The transformation is

$$
\begin{aligned}
& \sum_{l=0}^{\infty} \frac{\left(A^{1 / 3} ; q^{1 / 3}\right)_{l}\left(1-A^{1 / 3} q^{2 l / 3}\right)}{\left(q^{1 / 3} ; q^{1 / 3}\right)_{l}\left(1-A q^{2 l}\right)}\left(A^{1 / 3} q^{1 / 3} x\right)^{l} \\
& \quad=\frac{(A x)_{\infty}}{(x)_{\infty}} \sum_{k=0}^{\infty} \frac{\left(A^{1 / 3} ; q^{1 / 3}\right)_{3 k+1} q^{-\left(\begin{array}{l}
k \\
2
\end{array}\right)}(-x)^{k}}{(q)_{k}\left(1-A q^{2 k}\right)(A x)_{k}(1-x / q) \cdots\left(1-x / q^{k}\right)}
\end{aligned}
$$

The dual evaluation is [6, Equation (4.5)], namely,

$$
\begin{gathered}
\sum_{k=0}^{n} \frac{\left(q^{-n}\right)_{k}\left(A q^{n}\right)_{k}\left(A^{1 / 3} q^{1 / 3} ; q^{1 / 3}\right)_{3 k} q^{k}}{(q)_{k}(q A)_{2 k}} \\
=\frac{(q)_{n}\left(A^{1 / 3} q^{1 / 3} ; q^{1 / 3}\right)_{n-1}\left(1-A^{1 / 3} q^{2 n / 3}\right)(A q)^{n / 3}}{\left(q^{1 / 3} ; q^{1 / 3}\right)_{n}(A q)_{n-1}\left(1-A q^{2 n}\right)}
\end{gathered}
$$

For [6, Equation (4.8)] we take

$$
f_{l}= \begin{cases}0, & l \neq 0(\bmod 3), \\ \frac{\left(A ; q^{3}\right)_{L}}{\left(q^{3} ; q^{3}\right)_{L}} A^{L}, & l=3 L\end{cases}
$$

Again, (4.7) with base $q^{3}$ implies

$$
a_{k}=\frac{\left(A ; q^{3}\right)_{k}}{(q)_{k}} q^{\left({ }^{k+1}\right)}(-1)^{k}
$$


The transformation is

$$
\sum_{k=0}^{\infty} \frac{\left(A ; q^{3}\right)_{l}}{\left(q^{3} ; q^{3}\right)_{l}}\left(A x^{3}\right)^{l}=\frac{(A x)_{\infty}}{(x)_{\infty}} \sum_{k=0}^{\infty} \frac{\left(A ; q^{3}\right)_{k} q^{-\left(\begin{array}{c}
k \\
2
\end{array}\right)}(-x)^{k}}{(q)_{k}(A x)_{k}(1-x / q) \cdots\left(1-x / q^{k}\right)} .
$$

However, the left-hand side of (4.29) can be evaluated by the $q$-binomial theorem to obtain

$$
\sum_{k=0}^{\infty} \frac{\left(A ; q^{3}\right)_{k} q^{-\left(\begin{array}{l}
k \\
2
\end{array}\right)(-x)^{k}}}{(q)_{k}(A x)_{k}(1-x / q) \cdots\left(1-x / q^{k}\right)}=\frac{\left(A^{2} x^{3} ; q^{3}\right)_{\infty}(x)_{\infty}}{\left(A x^{3} ; q^{3}\right)_{\infty}(A x)_{\infty}} .
$$

The above equation is the $q$-analog of

$$
\left(1+3 x /(1-x)^{2}\right)^{-a}=(1-x)^{3 a}\left(1-x^{3}\right)^{-a} \text {. }
$$

We had previously stated (4.30) in [24, Equation (6.7)] and commented that it should be related to a $q$-Lagrange inversion proof of

$$
\sum_{k=0}^{\infty} \frac{\left(q^{-n}\right)_{k}\left(A q^{n}\right)_{k}\left(A ; q^{3}\right)_{k}}{(q)_{k}(A)_{2 k}} q_{k}= \begin{cases}0, & n \neq 0(\bmod 3) \\ \frac{\left(A ; q^{3}\right)_{N}(q)_{3 N}}{(A)_{3 N}\left(q^{3} ; q^{3}\right)_{N}} A^{N}, & n=3 N\end{cases}
$$

Indeed (4.32) is the dual evaluation.

We give only one more example in this section. As Andrews [6] has indicated, there are other examples involving only $q$. They could quite possibly lead to interesting transformations. Also, we could give the "derivative form" of Theorem 3.15 for each transformation in this section. We combine these two possibilities into one example. Andrews [6, Equation (4.9)] allows

$$
\begin{gathered}
a_{k}=\frac{q^{\left(\frac{k}{2}\right)+k}(-1)^{k}}{\left(1-q^{2 k+1}\right)}, \\
(-1)^{k} f_{k}= \begin{cases}q^{\left(3 n^{2}+7 n-2\right) / 2}, & k=3 n-1, \\
q^{\left(3 n^{2}-n\right) / 2}, & k=3 n, \\
-\left(1+q^{2 n+1}\right) q^{\left(3 n^{2}+7 n+2\right) / 2}, & k=3 n+1,\end{cases}
\end{gathered}
$$

and $A=q$, in Theorem 3.15. The transformation is

$$
\begin{aligned}
\sum_{n=1}^{\infty}(-1)^{n+1} & q^{\left(3 n^{2}+7 n-2\right) / 2} x^{3 n-1}+\sum_{n=0}^{\infty}(-1)^{n} q^{\left(3 n^{2}-n\right) / 2} x^{3 n} \\
& +\sum_{n=0}^{\infty}(-1)^{n}\left(1+q^{2 n+1}\right) q^{\left(3 n^{2}+7 n+2\right) / 2} x^{3 n+1} \\
= & \frac{\left(1-q^{2} x^{2}\right)}{(1-x)} \sum_{k=0}^{\infty} \frac{q^{-\left(\frac{k}{2}\right)}(-x)^{k}}{(q x)_{k+2}(1-x / q) \cdots\left(1-x / q^{k}\right)}
\end{aligned}
$$


Equation (4.35) is the $q$-analog of

$$
\frac{(1+x)^{2}}{1+x^{3}}=\frac{(1+x)}{(1-x)^{2}\left(1+x /(1-x)^{2}\right)}
$$

5. More tranformations. In $\S 4$ we carefully considered Theorem 3.2 . In this section we find transformations and evaluations for Theorem 3.3.

For Theorem 3.3 we are trying to evaluate

$$
f_{n}=\frac{(A)_{n}}{(q)_{n}} \sum_{k=0}^{n} \frac{\left(q^{-n}\right)_{k}}{(A)_{k}}(-1)^{k} q^{-\left(\begin{array}{c}
k \\
2
\end{array}\right)} a_{k} .
$$

So any evaluation in which the terminating parameter occurs exactly once will give a transformation. It is easy to see that the terminating ${ }_{2} \Phi_{1}$ evaluation and

$$
a_{k}=\frac{(A)_{k}(B)_{k}}{(q)_{k}(C)_{k}} q^{\left(\frac{k}{2}\right)+k}(-1)^{k}
$$

imply

$$
f_{n}=\frac{(A)_{n}(B)_{n}}{(q)_{n}(C)_{n}} B^{n}
$$

The resulting transformation is the $q$-analog of the linear ${ }_{2} F_{1}$ tranformation [31]. A nontrivial result is obtained if we take

$$
a_{k}=\frac{(A)_{k}\left(C ; q^{2}\right)_{k}}{(q)_{k}(C ; q)_{k}} q^{\left(\frac{k}{2}\right)+k}(-1)^{k}
$$

and use a $q$-analog of a terminating ${ }_{2} F_{1}(2)$ evaluation [7, Equation (1.8)] to find

$$
f_{n}= \begin{cases}0, & n \text { odd }, \\ \frac{(A)_{2 N} C^{N}}{\left(q^{2} ; q^{2}\right)_{N}\left(C q ; q^{2}\right)_{N}}, & n=2 N \text { even } .\end{cases}
$$

The tranformation is

$$
{ }_{2} \Phi_{1}\left[\begin{array}{c}
A, A q \\
C q
\end{array} \mid q^{2} ; C x^{2}\right]=\frac{(A x)_{\infty}}{(x)_{\infty}} \sum_{k=0}^{\infty} \frac{(A)_{k}\left(C ; q^{2}\right)_{k} q^{-\left(\frac{k}{2}\right)}(-x)^{k}}{(q)_{k}(C)_{k}(1-x / q) \cdots\left(1-x / q^{k}\right)}
$$

This is the $q$-analog of Equation (17) in [20,p. 112]. Jain [27, Equation (3.1)] has previously stated it. The dual evaluation follows from the $q$-analog of Vandermonde's theorem. In fact, it can be used to give another transformation. Put

$$
a_{k}= \begin{cases}0, & k \text { odd }, \\ \frac{\left.(A)_{2 K} q^{\left(2 K_{2}+1\right.}\right)}{\left(q^{2} ; q^{2}\right)_{K}\left(C q ; q^{2}\right)_{K}}, & k=2 K \text { even }\end{cases}
$$

so

$$
f_{n}=\frac{(A)_{n}\left(C ; q^{2}\right)_{n}}{(q)_{n}(C)_{n}} q^{-\left(\begin{array}{c}
n \\
2
\end{array}\right)}
$$


and

$$
\begin{aligned}
& \sum_{n=0}^{\infty} \frac{(A)_{n}\left(C ; q^{2}\right)_{n}}{(q)_{n}(C)_{n}} q^{-\left(\begin{array}{l}
n \\
2
\end{array}\right)} x^{n} \\
& \quad=\frac{(A x)_{\infty}}{(x)_{\infty}} \sum_{k=0}^{\infty} \frac{(A)_{2 k} q^{-\left(\begin{array}{c}
2 k \\
2
\end{array}\right)} x^{2 k}}{\left(q^{2} ; q^{2}\right)_{k}\left(C q ; q^{2}\right)_{k}(1-x / q) \cdots\left(1-x / q^{2 k}\right)}
\end{aligned}
$$

The $q \rightarrow 1$ limit of (5.9) is Equation (4) of [20, p. 111],

$$
{ }_{2} F_{1}\left[\begin{array}{c}
a, c / 2 \\
c
\end{array} \mid 2 x\right]=(1-x)_{2}^{-a} F_{1}\left[\begin{array}{c|c}
a / 2,(a+1) / 2 & \frac{x^{2}}{(1-x)^{2}}
\end{array}\right] .
$$

We can also take

$$
a_{k}=\frac{(A)_{k} q^{\left(\frac{k}{2}\right)+k / 2}(-1)^{k}}{\left(q^{1 / 2} ; q^{1 / 2}\right)_{k}\left(-C ; q^{1 / 2}\right)_{k}}
$$

to find

$$
f_{n}=\frac{(A)_{n}\left(C ; q^{1 / 2}\right)_{2 n}}{(q)_{n}\left(C^{2}\right)_{n}} q^{-n^{2} / 2}(-1)^{n}
$$

Thus

$$
\begin{aligned}
& \sum_{n=0}^{\infty} \frac{(A)_{n}\left(C ; q^{1 / 2}\right)_{2 n}}{(q)_{n}\left(C^{2}\right)_{n}} q^{-n^{2} / 2}(-x)^{n} \\
& \quad=\frac{(A x)_{\infty}}{(x)_{\infty}} \frac{(A)_{k} q^{-\left({ }_{2}^{2+1}\right)}\left(-x q^{1 / 2}\right)^{k}}{\left(q^{2} ; q^{2}\right)_{k}\left(-C ; q^{1 / 2}\right)_{k}(1-x / q) \cdots\left(1-x / q^{k}\right)}
\end{aligned}
$$

The dual evaluation is

$$
\sum_{l=0}^{k} \frac{\left(q^{-k}\right)_{l}\left(C ; q^{1 / 2}\right)_{2 l}}{(q)_{l}\left(C^{2}\right)_{l}} q^{l k-l^{2} / 2}(-1)^{l}=\frac{\left(-q^{1 / 2} ; q^{1 / 2}\right)_{k}}{\left(-C ; q^{1 / 2}\right)_{k}} q^{-k / 2} .
$$

By writing (5.14) on base $q^{-1}$, changing $C^{-1}$ to $C$ and $q^{-1}$ to $q$ we see that (5.14) really is

$$
{ }_{3} \Phi_{2}\left[\begin{array}{c}
q^{-k}, C, C q^{1 / 2} \mid q ; q \\
C^{2}, 0
\end{array}\right]=\frac{\left(-q^{1 / 2} ; q^{1 / 2}\right)_{k}}{\left(-C ; q^{1 / 2}\right)_{k}} C^{k} .
$$

Equation (5.15) is not new. The ${ }_{3} \Phi_{2}$ can be transformed to a well-poised ${ }_{2} \Phi_{1}$. Nevertheless, we can use (5.15) to choose

$$
a_{k}=\frac{(A)_{k}\left(C ; q^{1 / 2}\right)_{2 k} q^{\left(\frac{k}{2}\right)+k}}{(q)_{k}\left(C^{2}\right)_{k}}(-1)^{k}
$$

and

$$
f_{n}=\frac{(A)_{n} C^{n}}{\left(q^{1 / 2} ; q^{1 / 2}\right)_{n}\left(-C ; q^{1 / 2}\right)_{n}} .
$$


The transformation is

$$
{ }_{2} \Phi_{1}\left[\begin{array}{c}
A^{1 / 2},-A^{1 / 2} \\
-C
\end{array} \mid q^{1 / 2} ; C x\right]=\frac{(A x)_{\infty}}{(x)_{\infty}} \sum_{k=0}^{\infty} \frac{(A)_{k}\left(C ; q^{1 / 2}\right)_{2 k} q^{-\left(\begin{array}{c}
k \\
2
\end{array}\right)}(-x)^{k}}{(q)_{k}\left(C^{2}\right)_{k}(1-x / q) \cdots\left(1-x / q^{k}\right)}
$$

The appropriate $q \rightarrow 1$ limits of (5.13) and (5.18) are factorizations involving the binomial theorem.

One may feel that the $q^{-\left(\begin{array}{l}n \\ 2\end{array}\right)}$ factor does not belong in (5.9). In fact, writing the factors on base $q^{-1}$ will eliminate this term. We would like to change $q^{-1}$ to $q$ throughout (5.9). This process is justified by Theorem 3.13. The resulting transformation is

$$
\sum_{n=0}^{\infty} \frac{(A)_{n}\left(B ; q^{2}\right)_{n}}{(q)_{n}(B)_{n}} y^{n}=\frac{(A y)_{\infty}}{(y)_{\infty}} \sum_{k=0}^{\infty} \frac{(A)_{2 k} q^{4\left(\begin{array}{c}
k \\
2
\end{array}\right)+k}\left(B y^{2}\right)^{k}}{\left(q^{2} ; q^{2}\right)_{k}\left(B q ; q^{2}\right)_{k}(A y)_{2 k}} .
$$

We could change any of the transformations of $\$ \S 4$ and 5 in this way.

6. More evaluations. Finally we come to Theorems 3.1 and 3.4-3.6. In these cases we cannot sum $G_{k}(x)$, so that the resulting transformations are less interesting. Hence, we concentrate on the evaluations. In particular we give $q$-analogs of the strange evaluations (1.2)-(1.5), (1.7), and (1.8) of [24].

First, for Theorem 3.4 if we put

$$
a_{k}=\frac{(B)_{k}\left(A^{2} q^{1 / 2} / B\right)_{k}}{(q)_{k}\left(A q^{1 / 2} ; q^{1 / 2}\right)_{k}} q^{\left(\frac{k}{2}\right)+k}(-1)^{k},
$$

then the $q$-analog of Saalschütz' theorem implies

$$
f_{n}=\frac{\left(B / A ; q^{1 / 2}\right)_{n}\left(A q^{1 / 2} / B ; q^{1 / 2}\right)_{n}}{(q)_{n}\left(A q^{1 / 2} ; q^{1 / 2}\right)_{n}}(-A)^{n} q^{-1 / 2\left(\begin{array}{c}
n \\
2
\end{array}\right)}
$$

The dual evaluation is

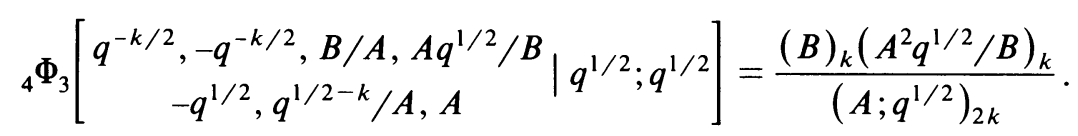

This is the $c=q^{-k}$ terminating version of the $q$-analog of Whipple's theorem [5, Theorem 2]. If $A=q^{a / 2}$, and $q \rightarrow 1$, then $G_{k}(x) \rightarrow x^{k}(1+x / 2)^{a+k}$. So our transformation is a $q$-analog of Equation (22) in [20, p. 112],

$$
\begin{aligned}
{ }_{2} F_{1}\left[\begin{array}{c}
b-a \\
1+a
\end{array}\right. & \left.1+a-b \mid \frac{-x}{2}\right] \\
& =(1+x / 2){ }_{2}^{a} F_{1}\left[\begin{array}{c}
b / 2, a+(1-b) / 2 \\
1+a
\end{array} \mid-2 x(1+x / 2)\right] .
\end{aligned}
$$

Next, we take Theorem 3.1. If $A=q^{a / 2}$ and $q \rightarrow 1$, then

$$
G_{k}(x) \rightarrow x^{k}(1-x / 2)^{-a-3 k} \text {. }
$$


So any pair $\left(f_{n}, a_{k}\right)$ gives a $q$-analog of a cubic transformation. We see that

$$
f_{n}=\frac{\left(A ; q^{1 / 2}\right)_{n}}{(q)_{n}} \sum_{k=0}^{n} \frac{\left(A q^{n / 2} ; q^{1 / 2}\right)_{2 k}\left(q^{-n}\right)_{k}}{\left(A ; q^{1 / 2}\right)_{3 k}} q^{-\left(\begin{array}{l}
k \\
2
\end{array}\right)}(-1)^{k} a_{k} .
$$

Choosing

$$
a_{k}=\frac{\left(A ; q^{1 / 2}\right)_{3 k} q^{\left(\frac{k}{2}\right)+k}(-1)^{k}}{(q)_{k}(C)_{k}\left(A^{2} q^{3 / 2} / C\right)_{k}}
$$

again we find

$$
f_{n}=\frac{\left(A ; q^{1 / 2}\right)_{n}\left(A q / C ; q^{1 / 2}\right)_{n}\left(C / A q^{1 / 2} ; q^{1 / 2}\right)_{n}}{(q)_{n}(C)_{n}\left(A^{2} q^{3 / 2} / C\right)_{n}} q^{1 / 2\left(\begin{array}{c}
n+1 \\
2
\end{array}\right)} A^{n} .
$$

The dual evaluation is

$$
\begin{gathered}
\sum_{k=0}^{n} \frac{\left(q^{-n}\right)_{k}\left(A ; q^{1 / 2}\right)_{k}\left(A q / C ; q^{1 / 2}\right)_{k}\left(C / A q^{1 / 2} ; q^{1 / 2}\right)_{k}\left(1-A q^{3 k / 2}\right)}{\left(A q^{n+1 / 2} ; q^{1 / 2}\right)_{k}(q)_{k}(C)_{k}\left(A^{2} q^{3 / 2} / C\right)_{k}(1-A)} q^{n k+1 / 2\left({ }_{2}^{k+1}\right)} A^{k} \\
=\frac{\left(A q^{1 / 2} ; q^{1 / 2}\right)_{2 n}}{(C)_{n}\left(A^{2} q^{3 / 2} / C\right)_{n}} .
\end{gathered}
$$

This is the $q$-analog of the strange ${ }_{5} F_{4}(1 / 4)$ evaluation [24, Equation (1.9)]. The transformation is a $q$-analog of [12, Equation (4.05)],

$$
\begin{aligned}
& { }_{3} F_{2}\left[\begin{array}{c}
\left.a, a+2-2 c, 2 c-a-1 \mid \frac{x}{8}\right] \\
c, a-c+3 / 2
\end{array}\right. \\
& \quad=(1-x / 2)^{-a}{ }_{3} F_{2}\left[\begin{array}{c}
a / 3, a+1 / 3, a+2 / 3 \\
c, a-c+3 / 2
\end{array} \mid \frac{-27 x}{8(1-x / 2)^{3}}\right] .
\end{aligned}
$$

Just as we derived Watson's tranformation from (4.7), we can derive the $q$-analog of the strange ${ }_{7} F_{6}(1)$ evaluation [24, Equation (1.7)] from (6.8). This time take

$$
C_{n k}=\frac{\left(q^{-n}\right)_{k}\left(A D^{2} q^{n+1 / 2}\right)_{k}\left(A / D^{2}\right)_{k} A^{-k} q^{-1 / 2\left(\begin{array}{c}
k \\
2
\end{array}\right)}}{\left(A q^{n+1 / 2} ; q^{1 / 2}\right)_{k}\left(q^{-n} / D^{2} ; q^{1 / 2}\right)_{k}\left(D^{2} q^{1 / 2} ; q^{1 / 2}\right)_{k}} \frac{\left(1-A q^{3 k / 2}\right)}{(1-A)} .
$$

Again, in $\sum_{k=0}^{n} C_{n k} f_{k}=\sum_{j, k} C_{n k} B_{k j} a_{j}$, the $k$-sum can be evaluated by the reversal of (6.8). The result is precisely (1.4).

For Theorem 3.6 we see that if $A=q^{a}, G_{k}(x) \rightarrow x^{k}(1-2 x)^{-a-3 k / 2}$. So we can find $q$-analogs of (1.3) and (1.8) in [24]. This time

$$
a_{k}= \begin{cases}0, & k \text { odd, } \\ \frac{(A)_{3 K} q^{1 / 2\left({ }_{2}^{2 K}\right)+K}}{(q)_{K}(B)_{K}\left(A q^{3 / 2} / B\right)_{K}}, & k=2 K \text { even, }\end{cases}
$$

yields

$$
f_{n}=\frac{(A)_{n}\left(B q^{-1 / 2}\right)_{n}(q A / B)_{n} q^{-1 / 2\left(\begin{array}{l}
n \\
2
\end{array}\right)}}{\left(q^{1 / 2} ; q^{1 / 2}\right)_{n}\left(B q^{-1 / 2} ; q^{1 / 2}\right)_{n}\left(q A / B ; q^{1 / 2}\right)_{n}}
$$


The dual evaluation is

$$
\begin{gathered}
\sum_{k=0}^{n} \frac{\left(q^{-n / 2} ; q^{1 / 2}\right)_{k}(A)_{k}\left(B q^{-1 / 2}\right)_{k}(q A / B)_{k}\left(1-A q^{3 k / 2}\right)}{\left(q^{1 / 2} ; q^{1 / 2}\right)_{k}\left(A q^{1+n / 2}\right)_{k}\left(B q^{-1 / 2} ; q^{1 / 2}\right)_{k}\left(q A / B ; q^{1 / 2}\right)_{k}(1-A)} q^{\left[n k-\left(\begin{array}{l}
k \\
2
\end{array}\right)\right] / 2} \\
= \begin{cases}0, & n \text { odd }, \\
\frac{(A q)_{N}\left(q^{1 / 2}\right)_{N}}{(B)_{N}\left(A q^{3 / 2} / B\right)_{N}}, & n=2 N \text { even. }\end{cases}
\end{gathered}
$$

This is the $q$-analog of (1.3) in [24]. To boost (6.13) to the $q$-analog of (1.8), a strange ${ }_{7} F_{6}(1)$ evaluation, we reason as before. The result is

$$
\begin{aligned}
\sum_{k=0}^{n} \frac{\left(q^{-n / 2} ; q^{1 / 2}\right)_{k}\left(q A / F ; q^{1 / 2}\right)_{k}\left(F q^{(n-1) / 2} ; q^{1 / 2}\right)_{k}(A)_{k}(q A / B)_{k}\left(B q^{-1 / 2}\right)_{k}}{\left(A q^{1+n / 2}\right)_{k}(F)_{k}\left(A q^{(3-n) / 2} / F\right)_{k}\left(q^{1 / 2} ; q^{1 / 2}\right)_{k}\left(B q^{-1 / 2} ; q^{1 / 2}\right)_{k}\left(q A / B ; q^{1 / 2}\right)_{k}} \\
\cdot \frac{\left(1-A q^{3 k / 2}\right)}{(1-A)} q^{k / 2} \\
= \begin{cases}0, & n \text { odd }, \\
\frac{(A q)_{N}\left(F q^{1 / 2} / B\right)_{n}(B F / A q)_{N}\left(q^{1 / 2}\right)_{N}}{(F)_{N}\left(A q^{3 / 2} / B\right)_{N}(B)_{N}\left(F q^{-1 / 2} / A\right)_{N}}, & n=2 N \text { even. }\end{cases}
\end{aligned}
$$

The series in (6.14) is very similar to (1.4) in that it looks like a bibasic well-poised series. It is better not to write out all of the factors in base $q^{1 / 2}$. Finally, the transformation is a $q$-analog of [12, Equation (4.06)],

$$
\begin{aligned}
& { }_{3} F_{2}\left[\begin{array}{c}
a, b-1 / 2,1+a-b \\
2 b-1,2+2 a-2 b
\end{array} \mid 8 x\right] \\
& \quad=(1-2 x)^{-a}{ }_{3} F_{2}\left[\begin{array}{c}
a / 3, a+1 / 3, a+2 / 3 \\
b, a-b+3 / 2
\end{array} \frac{27 x^{2}}{(1-2 x)^{3}}\right] .
\end{aligned}
$$

In [24] we gave three strange ${ }_{3} F_{2}(3 / 4)$ evaluations. Andrews [6] gave the $q$-analog of one of them, (1.1), and we proved it in (4.30). The other two, (1.4) and (1.5), follow from Theorem 3.5. Here, if $A=q^{a / 3}$ then $G_{k}(x) \rightarrow x^{k}(1+x / 3)^{a+2 k}$. We see that

$$
f_{n}=\frac{\left(A q^{(1-n) / 3} ; q^{1 / 3}\right)_{n}}{(q)_{n}} \sum_{k=0}^{n} \frac{\left(q^{-n}\right)_{k}\left(A q^{1 / 3} ; q^{1 / 3}\right)_{2 k}}{\left(A q^{(1-n) / 3} ; q^{1 / 3}\right)_{3 k}} q^{-\left(\begin{array}{l}
k \\
2
\end{array}\right)}(-1)^{k} a_{k}
$$

or

$$
f_{n}=\sum_{k=0}^{n} \frac{\left(A q^{2 n / 3} ; q^{-1 / 3}\right)_{3 k} q^{n k}}{\left(A q^{2 n / 3} ; q^{-1 / 3}\right)_{2 k}(q)_{k}} a_{n-k} .
$$

From (6.17) the choice $A=q^{1 / 3}$ and

$$
a_{k}=\frac{(B)_{k}(q / B)_{k}}{\left(q^{2 / 3} ; q^{1 / 3}\right)_{2 k}}(-1)^{k} q^{\left({ }_{2}^{+1}\right)}
$$


gives (by the $q$-analog of Saalschütz' theorem)

$$
f_{n}=\frac{(B)_{n}(q / B)_{n}\left(B q^{(n-2) / 3} ; q^{1 / 3}\right)_{2 n}}{\left(q^{2 / 3} ; q^{1 / 3}\right)_{2 n}\left(B q^{n-1} ; q^{-1}\right)_{2 n}}(-1)^{n} q^{\left(\begin{array}{c}
n+1 \\
2
\end{array}\right)}
$$

The dual evaluation is equivalent to

$$
\sum_{k=0}^{n} \frac{\left(q^{-n}\right)_{k}\left(B q^{-1 / 3} ; q^{1 / 3}\right)_{k}\left(q^{2 / 3} / B ; q^{1 / 3}\right)_{k}}{\left(q^{1 / 3} ; q^{2 / 3}\right)_{2 k}\left(q^{-n} ; q^{1 / 3}\right)_{k}} q^{k / 3}=\frac{(B)_{n}(q / B)_{n}(q)_{n}}{\left(q^{1 / 3} ; q^{1 / 3}\right)_{3 n}}
$$

This is the $q$-analog of the ${ }_{3} F_{2}(3 / 4)$ in [24, Equation (1.4)]. The tranformation is a $q$-analog of

$$
{ }_{2} F_{1}\left[\begin{array}{c}
3 b-1,2-3 b \\
3 / 2
\end{array} \mid-\frac{x}{4}\right]=(1+x / 3)_{2} F_{1}\left[\begin{array}{c}
b, 1-b \\
3 / 2
\end{array} \mid \frac{-9 x(1+x / 3)^{2}}{4}\right]
$$

Another choice for (6.17) is $A=1$ and

$$
a_{k}=\frac{(B)_{k}\left(B^{-1}\right)_{k}}{\left(q^{1 / 3} ; q^{1 / 3}\right)_{2 k}}(-1)^{k} q^{\left(k_{2}^{k+1}\right)}
$$

Then we have

$$
f_{n}=\frac{(B)_{n}\left(B^{-1}\right)_{n}\left(B q^{(n-1) / 3} ; q^{-1 / 3}\right)_{2 n-1}}{\left(q^{1 / 3} ; q^{1 / 3}\right)_{2 n}\left(B q^{n-1} ; q^{-1}\right)_{2 n-1}} q^{\left({ }_{2}^{n+1}\right)}(-1)^{n} .
$$

The dual evaluation is equivalent to

$$
\sum_{k=0}^{n} \frac{\left(q^{-n}\right)_{k}\left(q^{1 / 3} B^{-1} ; q^{1 / 3}\right)_{k}\left(B q^{1 / 3} ; q^{1 / 3}\right)_{k}}{\left(q^{2 / 3} ; q^{1 / 3}\right)_{2 k}\left(q^{-n-1 / 3} ; q^{1 / 3}\right)_{k}} q^{k / 3}=\frac{(B q)_{n}\left(q B^{-1}\right)_{n}(q)_{n}}{\left(q^{2 / 3} ; q^{1 / 3}\right)_{3 n}}
$$

This is the $q$-analog of the ${ }_{3} F_{2}(3 / 4)$ in [24, Equation (1.5)]. The transformation is a $q$-analog of

$$
{ }_{2} F_{1}\left[\begin{array}{c}
3 b,-3 b \\
1 / 2
\end{array} \mid-\frac{x}{4}\right]={ }_{2} F_{1}\left[\begin{array}{c}
b,-b \\
1 / 2
\end{array} \mid \frac{-9 x(1+x / 3)^{2}}{4}\right] .
$$

For the dual form if we put

$$
f_{l}=\frac{q^{-\left(\frac{1}{2}\right)-l / 3}(-1)^{l}}{\left(q^{-1 / 3} ; q^{-1 / 3}\right)_{l}\left(A^{-1} ; q^{-1 / 3}\right)_{l}\left(1-A q^{2 l / 3}\right)}
$$

then

$$
a_{k}=\frac{\left(A^{3} q^{2 k-1} ; q^{-1}\right)_{k}(-1)^{k} q^{\left({ }_{2}^{2+1}\right)}}{\left(A ; q^{1 / 3}\right)_{2 k+1}(q)_{k}}
$$

The evaluation is

$$
\begin{aligned}
& \sum_{k=0}^{n} \frac{\left(q^{-n}\right)_{k}\left(A^{3}\right)_{2 k} q^{k}}{(q)_{k}\left(A^{3}\right)_{k}\left(A q^{(1-n) / 3} ; q^{1 / 3}\right)_{3 k}} \\
& =\frac{q^{2 n / 3-\left(\begin{array}{c}
n+1 \\
2
\end{array}\right)}(-1)^{n}(1-A)(q)_{n}}{\left(q^{-1 / 3} ; q^{-1 / 3}\right)_{n}\left(A^{-1} ; q^{-1 / 3}\right)_{n}\left(A q^{(1-n) / 3} ; q^{1 / 3}\right)_{n}\left(1-A q^{2 n / 3}\right)}
\end{aligned}
$$

This is an evaluation of a special balanced ${ }_{5} \Phi_{4}$. 
7. Identities of Rogers-Ramanujan type. It is well known that Watson's transformation implies the Rogers-Ramanujan identities,

$$
\sum_{k=0}^{\infty} \frac{q^{k^{2}}}{(q)_{k}}=\frac{1}{\left(q ; q^{5}\right)_{\infty}\left(q^{4} ; q^{5}\right)_{\infty}}
$$

and

$$
\sum_{k=0}^{\infty} \frac{q^{k^{2}+k}}{(q)_{k}}=\frac{1}{\left(q^{2} ; q^{5}\right)_{\infty}\left(q^{3} ; q^{5}\right)_{\infty}} .
$$

We proved Watson's transformation in §4. Because Rogers' technique was really Theorem 3.2, we can expect other identities of the Rogers-Ramanujan type to follow from the quadratic transformations in $\S 4$. In this section we give several examples.

In (4.13) replace $x$ by $x / A$ and let $A \rightarrow \infty$. We have

$$
\sum_{n=0}^{\infty} \frac{q^{2 n^{2}-n} x^{2 n}}{\left(q^{2} ; q^{2}\right)_{n}}=(x)_{\infty} \sum_{k=0}^{\infty} \frac{q^{\left(\frac{k}{2}\right)} x^{k}}{(q)_{k}(x)_{k}} .
$$

We see that (7.3) converges as a formal power series in $q$. Changing $q$ to $q^{2}$, replacing $x$ by $q$ and $q^{3}$, and using (7.1) and (7.2) we find

$$
\sum_{k=0}^{\infty} \frac{q^{k^{2}}}{(q)_{2 k}}=\frac{1}{\left(q ; q^{2}\right)_{\infty}\left(q^{4} ; q^{20}\right)_{\infty}\left(q^{16} ; q^{20}\right)_{\infty}}
$$

and

$$
\sum_{k=0}^{\infty} \frac{q^{k^{2}+2 k}}{(q)_{2 k+1}}=\frac{1}{\left(q ; q^{2}\right)_{\infty}\left(q^{8} ; q^{20}\right)_{\infty}\left(q^{12} ; q^{20}\right)_{\infty}}
$$

These are Rogers' identities of modulus 20 [31, Equations (79),(96)]. Another interesting choice in (7.3) is $x=-q$.

In (4.21) let $B \rightarrow \infty$, replace $x$ by $x / A$, let $A \rightarrow \infty$, replace $q$ by $q^{2}$ and $x$ by $-x q^{2}$ to find

$$
\sum_{l=0}^{\infty} \frac{q^{l^{2}} x^{l}}{(q)_{l}}=\left(-x q^{2} ; q^{2}\right)_{\infty} \sum_{k=0}^{\infty} \frac{q^{k^{2}} x^{k}}{\left(q^{2} ; q^{2}\right)_{k}\left(-q^{2} x ; q^{2}\right)_{k}} .
$$

This time the choices of $x=1$ and $q$ result in

$$
\sum_{k=0}^{\infty} \frac{q^{k^{2}}}{\left(q^{4} ; q^{4}\right)_{k}}=\frac{1}{\left(-q^{2} ; q^{2}\right)_{\infty}\left(q ; q^{5}\right)_{\infty}\left(q^{4} ; q^{5}\right)_{\infty}}
$$

and

$$
\sum_{k=0}^{\infty} \frac{q^{k^{2}+k}}{\left(q^{2} ; q^{2}\right)_{k}\left(-q^{3} ; q^{2}\right)_{k}}=\frac{1}{\left(-q^{3} ; q^{2}\right)_{\infty}\left(q^{2} ; q^{5}\right)_{\infty}\left(q^{3} ; q^{5}\right)_{\infty}} .
$$

These are Equations (20) and (17) of [31]. 
Another example arises from (5.19), the cousin of (5.9). If we replace $y$ by $y / A$, and let $A \rightarrow \infty$, the result is

$$
\sum_{n=0}^{\infty} \frac{\left(B ; q^{2}\right)_{n}}{(B ; q)_{n}(q)_{n}} q^{\left(\frac{n}{2}\right)}(-y)^{n}=(y)_{\infty} \sum_{k=0}^{\infty} \frac{q^{4 k^{2}-2 k}\left(B y^{2}\right)^{k}}{\left(q^{2} ; q^{2}\right)_{k}\left(B q ; q^{2}\right)_{k}(y)_{2 k}} .
$$

If $B \rightarrow \infty$ in (7.9), and we put $y=-q$ and $y=-q^{2}$, we find

$$
\sum_{k=0}^{\infty} \frac{q^{3 k^{2}}(-1)^{k}}{\left(q^{2} ; q^{2}\right)_{k}(-q)_{2 k}}=\frac{1}{(-q)_{\infty}\left(q ; q^{5}\right)_{\infty}\left(q^{4} ; q^{5}\right)_{\infty}}
$$

and

$$
\sum_{k=0}^{\infty} \frac{q^{3 k^{2}+2 k}(-1)^{k}}{\left(q^{2} ; q^{2}\right)_{k}\left(-q^{2}\right)_{2 k}}=\frac{1}{\left(-q^{2}\right)_{\infty}\left(q^{3} ; q^{5}\right)_{\infty}\left(q^{2} ; q^{5}\right)_{\infty}} .
$$

These are Equations (19) and (15) of [31]. If we use Equation 39 of [31],

$$
\sum_{k=0}^{\infty} \frac{q^{4 k^{2}}}{\left(q^{2} ; q^{2}\right)_{2 k}}=\frac{\left(-q^{10} ; q^{16}\right)_{\infty}\left(-q^{6} ; q^{16}\right)_{\infty}\left(q^{16} ; q^{16}\right)_{\infty}}{\left(q^{4} ; q^{4}\right)_{\infty}}
$$

and put $B=1$, and $y=-q$ in (7.9), we have

$$
1+\sum_{n=1}^{\infty} \frac{(-q)_{n-1}}{(q)_{n}} q^{\left({ }_{2}^{n+1}\right)}=\frac{(-q)_{\infty}\left(-q^{10} ; q^{16}\right)_{\infty}\left(-q^{6} ; q^{16}\right)_{\infty}\left(q^{16} ; q^{16}\right)_{\infty}}{\left(q^{4} ; q^{4}\right)_{\infty}}
$$

In a similar way, Equation (39) of [31],

$$
\sum_{k=0}^{\infty} \frac{q^{4 k^{2}+4 k}}{\left(q^{2} ; q^{2}\right)_{2 k+1}}=\frac{\left(-q^{14} ; q^{16}\right)_{\infty}\left(-q^{2} ; q^{16}\right)_{\infty}\left(q^{16} ; q^{16}\right)_{\infty}}{\left(q^{4} ; q^{4}\right)_{\infty}}
$$

and (7.9), with $B=q^{2}$ and $y=-q^{2}$, imply

$$
\sum_{n=0}^{\infty} \frac{(-q)_{n}}{(q)_{n+1}} q^{\left({ }^{n+1}\right)+n}=\frac{(-q)_{\infty}\left(-q^{14} ; q^{16}\right)_{\infty}\left(-q^{2} ; q^{16}\right)_{\infty}\left(q^{16} ; q^{16}\right)_{\infty}}{\left(q^{4} ; q^{4}\right)_{\infty}}
$$

We see that (7.15) and (7.13) are related to Equations (12) and (13) of [31], and they imply

$$
\begin{gathered}
\left(-q^{10} ; q^{16}\right)_{\infty}\left(-q^{6} ; q^{16}\right)_{\infty}\left(q^{16} ; q^{16}\right)_{\infty}-q\left(-q^{14} ; q^{16}\right)_{\infty}\left(-q^{2} ; q^{16}\right)_{\infty}\left(q^{16} ; q^{16}\right)_{\infty} \\
=(q)_{\infty}\left(-q^{2} ; q^{2}\right)_{\infty}
\end{gathered}
$$

This is given in Equation (64) of [31].

The same technique applied to (5.13) gives

$$
\sum_{n=0}^{\infty} \frac{q^{n^{2}+n / 2} x^{n}}{(q)_{n}}=(x q)_{\infty} \sum_{k=0}^{\infty} \frac{q^{\left(3 k^{2}+k\right) / 4} x^{k}}{\left(q^{1 / 2} ; q^{1 / 2}\right)_{k}(x q)_{k}} .
$$

This time the choices $x=q^{-1 / 2}$ and $x=q^{1 / 2}$ give

$$
\sum_{k=0}^{\infty} \frac{q^{\left(3 k^{2}-k\right) / 2}}{(q)_{k}\left(q ; q^{2}\right)_{k}}=\frac{1}{\left(q ; q^{2}\right)_{\infty}\left(q^{2} ; q^{10}\right)_{\infty}\left(q^{8} ; q^{10}\right)_{\infty}}
$$


and

$$
\sum_{k=0}^{\infty} \frac{q^{\left(3 k^{2}+3 k\right) / 2}}{(q)_{k}\left(q^{3} ; q^{2}\right)_{k}}=\frac{1}{\left(q^{3} ; q^{2}\right)_{\infty}\left(q^{4} ; q^{10}\right)_{\infty}\left(q^{6} ; q^{10}\right)_{\infty}}
$$

These are Equations (46) and (44) of [31]. The $x \rightarrow x / A, A \rightarrow \infty, B \rightarrow \infty$ limit of Sears' (4.6) is

$$
\sum_{k=0}^{\infty} \frac{q^{\left(\frac{k}{2}\right)}(-x)^{k}}{(q)_{k}(C)_{k}}=(x)_{\infty} \sum_{k=0}^{\infty} \frac{q^{\left(3 k^{2}-3 k\right) / 2}(-C x)^{k}}{(q)_{k}(C)_{k}(x)_{k}} .
$$

For $C=q^{1 / 2}$ and $x=-q^{1 / 2}$, again, we find (7.18) from (7.4). Also $C=q^{3 / 2}$ and $x=-q^{3 / 2}$ give (7.19) from (7.5). However $C=q^{1 / 2}, x=-q$, and Equation (99) of [31] imply

$$
\sum_{k=0}^{\infty} \frac{q^{3 k^{2}}}{(q)_{2 k}\left(-q^{2} ; q^{2}\right)_{k}}=\frac{\left(q ; q^{10}\right)_{\infty}\left(q^{9} ; q^{10}\right)_{\infty}\left(q^{8} ; q^{20}\right)_{\infty}\left(q^{12} ; q^{20}\right)_{\infty}\left(q^{10} ; q^{10}\right)_{\infty}}{\left(-q^{2} ; q^{2}\right)_{\infty}(q ; q)_{\infty}}
$$

Similarly, $C=q^{3 / 2}, x=-q$, and Equation (94) of [31] imply

$$
\sum_{k=0}^{\infty} \frac{q^{3 k^{2}+2 k}}{(q)_{2 k+1}\left(q^{2} ; q^{2}\right)_{k}}=\frac{\left(q^{3} ; q^{10}\right)_{\infty}\left(q^{7} ; q^{10}\right)_{\infty}\left(q^{16} ; q^{20}\right)_{\infty}\left(q^{4} ; q^{20}\right)_{\infty}\left(q^{10} ; q^{10}\right)_{\infty}}{\left(-q^{2} ; q^{2}\right)_{\infty}(q ; q)_{\infty}}
$$

These are given as Equations (100) and (97) of [31].

Yet another example is the $B \rightarrow B A^{1 / 2}, x \rightarrow x / A, A \rightarrow \infty$, and $q \rightarrow q^{2}$ case of (4.21). It is

$$
\sum_{l=0}^{\infty} \frac{q^{l^{2}} x^{l}}{(q)_{l}(q / B)_{l}}=\left(-x q^{2} ; q\right)_{\infty} \sum_{k=0}^{\infty} \frac{(-q / B)_{2 k} q^{k^{2}} x^{k}}{\left(q^{2} ; q^{2}\right)_{k}\left(q^{2} / B^{2} ; q^{2}\right)_{k}\left(-x q^{2} ; q^{2}\right)_{k}}
$$

The two pairs of choices $\left(B=q^{1 / 2}, x=q^{-1 / 2}\right)$ and $\left(B=q^{-1 / 2}, x=q^{1 / 2}\right)$ allow the evaluation of the right-hand side of $(7.23)$ as a limit of a ${ }_{2} \Phi_{1}$ on base $q^{2}$. The resulting evaluations of the left-hand side of (7.23) are Equations (85) and (84) of [31]. Conversely, the related evaluations Equations (39) and (38) allow ( $B=q^{1 / 2}$, $x=1)$ and $\left(B=q^{-1 / 2}, x=q\right)$ to find

$$
\sum_{k=0}^{\infty} \frac{\left(-q ; q^{2}\right)_{2 k} q^{2 k^{2}}}{\left(q^{8} ; q^{8}\right)_{k}\left(q^{2} ; q^{4}\right)_{k}}=\left(-q^{5} ; q^{8}\right)_{\infty}\left(-q^{3} ; q^{8}\right)_{\infty}\left(-q^{2} ; q^{2}\right)_{\infty}
$$

and

$$
\sum_{k=0}^{\infty} \frac{\left(q ; q^{2}\right)_{2 k+1} q^{2 k^{2}+2 k}}{\left(q^{2} ; q^{2}\right)_{2 k+1}\left(-q^{6} ; q^{4}\right)_{k}}=\frac{\left(-q ; q^{8}\right)_{\infty}\left(-q^{7} ; q^{8}\right)_{\infty}\left(q^{8} ; q^{8}\right)_{\infty}}{\left(q^{2} ; q^{2}\right)_{\infty}\left(-q^{6} ; q^{4}\right)_{\infty}}
$$

These two evaluations appear to be new.

We have not systematically tried to find such evaluations. We could also try the unstated cubic transformations of $\$ 5$ as double sums. Or we could use the Jacobi triple product identity as Slater did [31]. The derivative form of the transformation 
would insert a linear term, as in Theorem 3.15. Also we did not state any transformations with $q$ as the only parameter. In any case it is clear that identities of the Rogers-Ramanujan type are related to $q$-Lagrange inversion.

8. Connections with orthogonal polynomials. As Askey and Ismail [9] and Andrews [6] have indicated, Rogers found his identities by considering connection coefficient problems for certain orthogonal polynomials. These polynomials were $q$-analogs of Gegenbauer polynomials. In this section we show how this question is related to $q$-Lagrange inversion.

First we consider the case of $q=1$ and Jacobi polynomials,

$$
P_{n}^{(\alpha, \beta)}(x)=\frac{(\alpha+1)_{n}}{n !}{ }_{2} F_{1}\left[\begin{array}{c}
-n, n+\alpha+\beta+1 \\
\alpha+1
\end{array} \mid \frac{1-x}{2}\right] .
$$

If we let $r_{j}(x)=[(1-x) / 2]^{j}$, then we have

$$
P_{n}^{(\alpha, \beta)}(x)=\sum_{j=0}^{n} C_{n j} r_{j}(x)
$$

where

$$
C_{n j}=\frac{(\alpha+1)_{n}(\alpha+\beta+1)_{2 j}(-1)^{j}(\alpha+\beta+1+2 j)_{n-j}}{(\alpha+\beta+1)_{n}(\alpha+1)_{j} j !(n-j) !} .
$$

The inverse problem of expanding $r_{j}(x)$ in terms of $P_{n}^{(\alpha, \beta)}(x)$ is solved by finding $C_{j n}^{-1}$. We see that (2.7) and (2.8), for $b=1$ and $a=\alpha+\beta+1$, give

$$
C_{j k}^{-1}=\frac{(\alpha+\beta+1)_{k}(\alpha+1)_{j} j !(-\alpha-\beta-2 j)_{j-k-1}}{(\alpha+1)_{k}(\alpha+\beta+1)_{2 j}(-1)^{j}(j-k) !}(-\alpha-\beta-1-2 k) .
$$

This solves the connection coefficient problem of $P_{m}^{(\gamma, \delta)}(x)$ to $P_{n}^{(\alpha, \beta)}(x)$, because $P_{m}^{(\gamma, \delta)}(x)$ is given as a linear combination of $r_{j}(x)$.

For the $q$-Jacobi polynomials that are given in [8],

$$
P_{n}(x ; a, b ; q)={ }_{2} \Phi_{1}\left[\begin{array}{c}
q^{-n}, a b q^{n+1} \\
a q
\end{array} \mid q ; q x\right],
$$

we take $r_{j}(x)=x^{j}$. Again we see that we need to know the inverse of the matrix

$$
C_{n j}=\frac{\left(a b q^{1+2 j}\right)_{n-j}}{(q)_{n-j}} q^{-n j}
$$

This is given exactly in Theorem 3.2 with $A=a b q$. In fact, this argument works for the connection coefficients of the very general ${ }_{4} \Phi_{3}$ polynomials of Askey and Wilson [10]. The $n$-dependence for those polynomials is the same. Rogers' polynomials are special cases of the ${ }_{4} \Phi_{3}$ 's.

The Gegenbauer polynomials are the special case of Jacobi polynomials which have a quadratic transformation. So it is to be expected that a set of $q$-Gegenbauer polynomials should have a $q$-quadratic transformation. We have just seen that the quadratic transformations of $\$ 4$ imply several identities of the Rogers-Ramanujan type. It is not surprising then that Rogers could find these identities from $q$ Gegenbauer polynomials. 
It would be very interesting to find some orthogonal polynomials based upon our other examples of $q$-Lagrange inversion, e.g. Theorem 3.1. However, this is not even known for $q=1$. Some new identities of the Rogers-Ramanujan type might result.

9. Concluding remarks. F. H. Jackson [26] gave the first example of $q$-Lagrange inversion. It is a $q$-analog of Abel's generalization of the binomial theorem. His example is a special case of Theorem 3.7. Recently, J. Cigler [17, 18, 19] has also been finding other examples. It would be very interesting to incorporate any of these examples, or, for example, Theorem 1.2, into a general theory. For Theorem 1.2 the work of Gessel [23] and Garsia [22] would appear to be promising.

There are many directions open for the quadratic transformations. Verma and Jain have begun this program. We have indicated a systematic approach. However, the evaluations that we used were the standard ones-Vandermonde's theorem, Saalschütz' theorem, and the very well-poised ${ }_{6} \Phi_{5}$. It is quite possible that other evaluations could give other transformations [3, Equation (3.3)]. Verma and Jain have given several bibasic evaluations which might work [28]. In this regard, again, we caution that all transformations stated here are formal power series identities, and not identities for complex-valued functions. If the series do converge (or terminate), we could integrate against a $q$-beta function. This would boost the transformations to transformations of series of higher order. We have not done these calculations. They could quite possibly be related to [28].

We do not claim to have found all of the $q$-analogs of the matrices in $\$ 2$. It is possible that (2.9) gives a nontrivial matrix that is different from (2.10). Rather, we were interested in those values of $b$ which occurred in [24]. We wanted to find $q$-analogs of those strange evaluations. Other values for $b(\mathrm{e} . \mathrm{g} . p=0)$ could be interesting. Also, a basis for formal power series different from $\left\{x^{k}\right\}_{k=0}^{\infty}$ could be necessary for other matrices.

Perhaps the most intriguing part of this paper is the connection between $q$-Lagrange inversion and identities of the Rogers-Ramanujan type. In $\$ 4$ we indicated how Sears' quadratic transformation implies Watson's transformation and thus the Rogers-Ramanujan identities. Can one avoid Watson's transformation altogether, as we have in $\$ 7$ ? Bressoud [14] has shown how to derive these identities from terminating evaluations. For the Rogers-Ramanujan identities, he used the terminating ${ }_{6} \Phi_{5}$ evaluation. This is given exactly by $q$-Lagrange inversion on Sears' transformation. This indicates that our new evaluations in $\$ 6$ could lead to new identities of the Rogers-Ramanujan type. It is easy to modify the Bailey transform as given by Bressoud to fit $\S 6$, but we have not carried out any of these calculations.

The transformations implicit in $\$ 6$ could also give new identities, as in $\$ 7$. They are $q$-analogs of cubic transformations, written as double sums equal to single sums. We fould that an analog of Watson's transformation for a cubic transformation was the evaluation (1.4). A better analog might be a transformation of a double sum into a single sum. This could lead to double sum Rogers-Ramanujan identities, as in [28].

Another direction that we hope to pursue is the connection of the matrices with bilateral series. By extending the definition of $B_{n k}$ properly to negative values of $n$ and $k$, this can be done. 
Finally, it is possible to systematically study the transformations which arise just as Watson's theorem did in $\$ 4$. There we found the coefficient of $x^{n}$ in the product of a transformation and its derivative form. In fact, there is a general theorem that does this which is the $q$-analog of Theorem 2 in $[24, \S 4]$. We state one version here.

Theorem 9.1. Let $B_{n k}$ and $G_{k}(x)$ be defined as in Theorem 3.7. Put $H_{j}(x)=$ $\sum_{n=j}^{\infty} B_{n j}^{\prime} x^{n}$, where

$$
\left.B_{n j}^{\prime}=\frac{\left(B q^{-j} p^{-n+1} ; p\right)_{n-j-1}}{(q)_{n-j}}\left(1-B q^{-n} p^{-n}\right) q^{(n-j+1}\right)-n j(B / A)^{n \delta}(-1)^{n-j} .
$$

Suppose $\sum_{k=0}^{\infty} a_{k} G_{k}(x)=\sum_{k=0}^{\infty} b_{k} x^{k}$ and $\sum_{j=0}^{\infty} c_{j} H_{j}(x)=\sum_{j=0}^{\infty} d_{j} x^{j}$. Then, if $B=$ $A p^{n} q^{n}, p=q^{1 / b}$, and $\delta(1+1 / b)=1, \sum_{k=0}^{n} a_{k} c_{n-k} q^{k n}=\sum_{k=0}^{n} b_{k} d_{n-k}$.

Added IN Proof. A formula equivalent to Theorem 3.7 was given by L. Carlitz, Some inverse relations, Duke Math. J. 40 (1973), 893-901.

\section{REFERENCES}

1. N. Abel, Sur les fonctions génératrices et leurs déterminantes, Ouvres Complètes, vol. 2, Grøndahl \& Søn, Christiania, 1881, pp. 67-81.

2. , Beweis eines Ausdruckes, von welchem die Binomial-Formel ein einzelner Fall ist, J. Reine Angew. Math. 1 (1826), 159-160.

3. W. Al-Salam and A. Verma, On quadratic transformations for basic series, preprint.

4. G. Andrews, Identities in combinatorics. II: A q-analog of the Lagrange inversion theorem, Proc. Amer. Math. Soc. 53 (1975), 240-245.

5. , On q-analogues of the Watson and Whipple summations, SIAM J. Math. Anal. 7 (1976), 332-336.

6. Connection coefficient problems and partitions (D. Ray-Chaudhuri, ed.), Proc. Sympos. Pure Math., vol. 34, Amer. Math. Soc., Providence, R. I., 1979, pp. 1-24.

7. $\quad$ On the q-analog of Kummer's theorem and applications, Duke Math. J. 40 (1973), 525-528.

8. G. Andrews and R. Askey, Enumeration of partitions: The role of Eulerian series and q-orthogonal polynomials, Higher Combinatorics (M. Aigner, ed.), Reidel, Dordrecht, 1977, pp. 3-26.

9. R. Askey and M. Ismail, A generalization of ultraspherical polynomials, Studies in Analysis (to appear).

10. R. Askey and J. Wilson, Some basic hypergeometric orthogonal polynomials that generalize Jacobi polynomials, preprint.

11. W. Bailey, Generalized hypergeometric series, Cambridge Univ. Press, Cambridge, 1935.

12. __ Products of generalized hypergeometric series, Proc. London Math. Soc. 28 (1928), 242-254.

13. Some identities involving generalized hypergeometric series, Proc. London Math. Soc. 29 (1929), 503-516.

14. D. Bressoud, Some identities for terminating $q$-series, Math. Proc. Cambridge Philos. Soc. 89 (1981), 211-223.

15. L. Carlitz, Some q-expansion formulas, Glas. Mat. Ser. III 8 (1973), 205-214.

16. S_ Some formulas of F. H. Jackson, Monatsh. Math. 73 (1969), 193-198.

17. J. Cigler, Operatormethoden für q-Identitäten, Monatsh. Math. 88 (1980), 87-105.

18. __ Operatormethoden für q-Identitäten. II. q-Laquerre-Polynome, Monatsh. Math. 91 (1981), 105-117.

19. __ Operatormethoden für q-Identitäten. III: Umbrale Inversion und die Lagrangesche Formel, Arch. Math. (Basel) 35 (1980), 533-543.

20. A. Erdelyi, Higher transcendental functions, Vol. 1, McGraw-Hill, New York, 1953.

21. J. Fields and M. Ismail, Polynomial expansions, Math. Comp. 29 (1975), 894-902.

22. A. Garsia, A q-analogue of the Lagrange inversion formula, Houston J. Math. 7 (1981), 205-237.

23. I. Gessel, $A$ noncommutative generalization and q-analog of the Lagrange inversion formula, Trans. Amer. Math. Soc. 257 (1980), 455-482. 
24. I. Gessel and D. Stanton, Strange evaluations of hypergeometric series, SIAM J. Math. Anal. 13 (1982), 295-308.

25. P. Henrici, Applied and computational complex analysis, Vol. 1, Interscience, New York, 1977.

26. F. Jackson, A q-generalization of Abel's series, Rend. Palermo 29 (1910), 340-346.

27. V. Jain, Some transformations of basic hypergeometric functions. Part II, SIAM J. Math. Anal. 12 (1981), 957-961.

28. V. Jain and A. Verma, Transformations between basic hypergeometric series on different bases and identities of Rogers-Ramanujan type, J. Math. Anal. Appl. 76 (1980), 230-269.

29. D. Sears, Transformations of basic hypergeometric functions of special type, Proc. London Math. Soc. 52 (1951), 467-483.

30. L. Slater, Generalized hypergeometric functions, Cambridge Univ. Press, Cambridge, 1966.

31. __ Further identities of the Rogers-Ramanujan type, Proc. London Math. Soc. 54 (1952), 147-167.

32. A. Verma, A quadratic transformation of a basic hypergeometric series, SIAM J. Math. Anal. 11 (1980), 425-427.

33. E. Whittaker and G. Watson, A course of modern analysis, Cambridge Univ. Press, Cambridge, 1927.

Department of Mathematics, Massachusetts institute of Technology, Cambridge, MasSACHUSETTS 02139

School of Mathematics, University of Minnesota, Minneapolis, Minnesota 55455 